\title{
MARC POLZIN
}

\section{Prolongement de la valeur absolue de Gauss et problème de Skolem}

Bulletin de la S. M. F., tome 116, no 1 (1988), p. 103-132

<http://www.numdam.org/item?id=BSMF_1988_116_1_103_0>

(C) Bulletin de la S. M. F., 1988, tous droits réservés.

L'accès aux archives de la revue «Bulletin de la S. M. F. » (http: //smf.emath.fr/Publications/Bulletin/Presentation.html) implique l'accord avec les conditions générales d'utilisation (http://www.numdam.org/ conditions). Toute utilisation commerciale ou impression systématique est constitutive d'une infraction pénale. Toute copie ou impression de ce fichier doit contenir la présente mention de copyright.

\section{NumDam}




\title{
PROLONGEMENT DE LA VALEUR ABSOLUE DE GAUSS ET PROBLÈME DE SKOLEM
}

PAR

\author{
MARC POLZIN $(*)$
}

RÉSUMÉ. - Dans cet article nous nous intéressons au problème suivant : soit $\left(K,|\cdot|_{0}\right)$ un corps valué ultramétrique, non nécessairement complet, $L / K$ un corps de fonctions d'une variable sur $K,\left\{|\cdot|_{i}\right\}_{1 \leq i \leq s}$ des valeurs absolues sur $L$ qui prolongent $|\cdot|_{0}$, telles que le corps résiduel $\left(\overline{L,|\cdot|_{i}}\right)$ de $\left(L,|\cdot|_{i}\right)$ soit un corps de fonctions d'une variable sur $\left(\overline{K,|\cdot|_{0}}\right)$, le corps résiduel de $K$. Existe-t-il $T \in L$, transcendant sur $K$ tel que $\left\{|\cdot|_{i}\right\}_{1<i \leq s}$ soit exactement les prolongements à $L$ de la valeur absolue de Gauss sur $K(T)$ associée à $T$ et à $|\cdot|_{0}$ ? Nous donnons une condition équivalente en termes géométriques en utilisant la réduction analytique des courbes algébriques en géométrie analytique rigide (THÉORÈME 1) et nous montrons (THÉORĖME 2) comment ce problème est relié à un problème arithmétique étudié par Skolem.

ABSTRACT. - In this article we are interested with the following problem : let be $\left(K,|\cdot|_{0}\right)$ a valued field, non archimedean, not necesseraly complete, $L / K$ a function field of one variable over $K,\left\{|\cdot|{ }_{i}\right\}_{1 \leq i \leq s}$ a set of absolute values on $L$ which extend $|\cdot|_{0}$ and such that the residue field $\left(\overline{L,|\cdot|_{i}}\right)$ of $\left(L,|\cdot|_{i}\right)$ is a function field of one variable over $\left(\overline{K,\left.|\cdot|\right|_{0}}\right)$ the residue fields of $K$. Does it exist $T \in L$, transcendental over $K$ such that $\left\{|\cdot|_{i}\right\}_{1 \leq i \leq s}$ is exactly the set of all extensions to $L$ of the Gauss valuation on $K(T)$ associated to $T$ and $|\cdot|_{0}$ ? We give a geometrical equivalent condition, using analytical reduction of algebraic curves, by help of technics providing from rigid analytical geometry (TheOrem 1) and we show (ThEOREM 2) how this problem is connected with an arithmetical problem studied by Skolem.

\section{Introduction}

Soient $\left(K,|\cdot|_{0}\right)$ un corps muni d'une valeur absolue ultramétrique, $L / K$ un corps de fonctions d'une variable dont le corps des constantes est purement inséparable sur $K$. On s'intéresse aux valeurs absolues $|\cdot|$ sur $L$ qui prolongent $|\cdot|_{0}$ et telles que

\section{(A) $(\overline{L,|\cdot|}) /\left(\overline{K,|\cdot|_{0}}\right)$ soit un corps de fonctions à une variable}

$\left(^{*}\right)$ Texte reçu le 23 juillet 1986, révisé le 17 juin 1987.

M. Polzin, Université de Bordeaux I, Mathématiques et Informatique, 351 cours de la Libération, 33405 Talence Cedex, France

BULLETIN DE LA SOCIÉTÉ MATHÉMATIQUE DE FRANCE $\quad$ 0037-9484/1988/103/\$5.00 (C) Société mathématique de France 
où $(\overline{L,|\cdot|})$ (resp. $(\overline{K,|\cdot| 0})$ ) désigne le corps résiduel de $(L,|\cdot|)$ (resp. $(K,|\cdot| 0))$.

Une telle valeur absolue est caractérisée par le fait qu'il existe $T \in L$ tel que $|T|=1$ et que l'image résiduelle de $T$ soit transcendante sur $\bar{K}=\left(\overline{K,|\cdot|_{0}}\right)$; il suit de cela que $\left|\sum_{i} a_{i} T^{i}\right|=\max \left|a_{i}\right|$, on dit alors que $|\cdot|$ est la valeur absolue de Gauss sur $K(T)$ associée $\grave{a}|\cdot|_{0}$ et à $T$.

Soient $\left\{|\cdot|_{i}\right\}_{1 \leq i \leq s}$ des prolongements à $L$ de $|\cdot|_{0}$ satisfaisant (A). L'objet de plusieurs articles [De 1,2], [La1], [Ne], [Po] est d'établir une relation entre le genre de $L / K$ et les genres de $\left(\overline{L,|\cdot|_{i}}\right) /\left(\overline{K,|\cdot|_{0}}\right)$; ils obtiennent une relation sous la condition

(B) qu'il existe $T \in L$ transcendant sur $K$ tel que $\left\{|\cdot|_{i}\right\}_{1 \leq i \leq s}$ soient exactement les prolongements à $L$ de la valeur absolue de Gauss sur $K(T)$ associée $\grave{a} T$ et $\grave{a}|\cdot|_{0}$.

La question est donc de savoir si tout système de valeurs absolues satisfaisant $(\mathrm{A})$ satisfait $(\mathrm{B})$. La réponse est positive dans le cas où $\left(K,|\cdot|_{0}\right)$ est complet [Ma2, théorème 3]. En général la réponse est négative, ici nous donnons une condition (C), nécessaire et suffisante sur $\left\{\left(K,|\cdot|_{0}\right) ; L / K\right\}$ pour que tout système $\left\{|\cdot|_{i}\right\}_{1 \leq i \leq s}$ de valeurs absolues sur $L$ (satisfaisant (A)) satisfasse (B). Cette condition (C), que nous allons expliciter est appelée propriété de Skolem locale parce qu'elle est reliée au problème de Skolem.

Soit $\widehat{K}$ le complété de $\left(K,|\cdot|_{0}\right)$, alors le nilradical $\mathfrak{N}$ de $\widehat{K} \otimes_{K} L$ est premier, de plus $\widehat{K}$ et $L$ s'injectent canoniquement dans $\operatorname{Fr}\left(\widehat{K} \otimes_{K} L / \mathfrak{N}\right)$, le corps des fractions de $\widehat{K} \otimes_{K} L / \mathfrak{N}$ et que l'on note $L \widehat{K}$; ainsi $L \widehat{K} / \widehat{K}$ est un corps de fonctions d'une variable.

On dit que $\left\{\left(K,|\cdot|_{0}\right) ; L / K\right\}$ satisfait la propriété (C) si pour tout $f_{1}, f_{2}, \ldots, f_{r} \in L$ les propriétés i) et ii) suivantes sont équivalentes :

(C) i) Il existe un anneau de valuation discrète $R$ de $L \widehat{K} / \widehat{K}$ (i.e. un anneau de valuation discrète avec $\operatorname{Fr}(R)=L \widehat{K}$ et $R \supset \widehat{K}$ ) tel que $f_{i} \in R$ et que $\left|f_{i}(R)\right|_{0} \leq 1$, pour $1 \leq i \leq r$, où $f_{i}(R)$ désigne l'image de $f_{i}$ dans le corps résiduel $M$ de $R$ (qui est fini sur $\widehat{K}$ ) et où $|\cdot|_{0}$ désigne l'unique prolongement à $M$ de la valeur absolue de $\widehat{K}$,

ii) il existe un anneau de valuation discrète $S$ de $L / K$ ( i.e. un anneau de valuation discrète avec $\operatorname{Fr}(S)=L$ et $S \supset K)$ tel que $f_{i} \in S$ et que $f_{i}(S)$ soit entier sur $K^{0}$ pour $1 \leq i \leq r$, où $K^{0}$ est l'anneau de valuation de $\left(K,|\cdot|_{0}\right)$ et où $f_{i}(S)$ est l'image de $f_{i}$ dans le corps résiduel de $S$ qui est fini sur $K$.

TOME $116-1988-\mathrm{N}^{\circ} 1$ 
Par suite la condition (B) sera en particulier satisfaite dès que $\left(K,|\cdot|_{0}\right)$ sera hensélien. Par ailleurs les travaux de Rumely [Ru], Roquette [Ro2] et Moret-BAILly, SzPIRo [Mo-Ba, Szp], [Mo-Ba] montrent que la condition (C), donc (B) est assurée si $\left(K,|\cdot|_{0}\right)$ est un corps de valuation discrète dont le corps résiduel est algébrique sur un corps fini.

Terminons par quelques mots sur les démonstrations. L'équivalence de (B) et (C), i.e., le ThÉORÈme 2 du paragraphe 3 passe par une interprétation géométrique de $(\mathrm{B})$ en utilisant la réduction analytique des courbes algébriques en géométrie rigide, en particulier des résultats de [Fr-Ma], [Ma1] et [Ma2].

Je tiens à remercier ici J. Fresnel et M. MAtignon, sans l'aide et le soutien desquels cet article n'aurait pu aboutir à son terme.

\section{Corps de fonctions valués, courbes, réduction analytique}

\subsection{Réduction des espaces analytiques, analytification des variétés algébriques.}

1.1.1. Réduction des espaces analytiques [Bo2], [Fr], [Fr, vdP]. - Dans tout ce paragraphe $K$ désigne un corps valué complet. Soient $A$ une $K$ algèbre affinoïde, $\|\cdot\|^{\text {sp }}$ la semi-norme spectrale sur $A$,

$$
A^{0}=\left\{f \in A ;\|f\|^{\text {sp }} \leq 1\right\}, \quad A^{00}=\left\{f \in A ;\|f\|^{\text {sp }}<1\right\}, \quad \bar{A}=A^{0} / A^{00},
$$

alors $\bar{A}$ est une $\bar{K}$-algèbre de type fini, réduite. L'application

$$
r: X=\operatorname{Spm} A \longrightarrow \bar{X} \stackrel{c}{\stackrel{\text { déf }}{=} \operatorname{Spm} \bar{A}}
$$

définie par $r(\mathfrak{M})=s\left(\sqrt{\mathfrak{M} \cap A^{0}+A^{00}}\right)$ (où $s: A^{0} \rightarrow \bar{A}$ est la surjection canonique) est surjective. On dit que $r: X \rightarrow \bar{X}^{c}$ est la réduction canonique de l'affinoïde $X$. Une partie $U$ de $X$ est appelée ouvert pur (ou formel) de $X$ si $U=r^{-1}(W)$ où $W$ est un ouvert (de Zariski) de $\bar{X}^{c}$; un ouvert formel est en particulier une réunion finie de parties rationnelles (donc affinoïdes) de $X$.

Une $K$-algèbre affinoïde $A$ est distinguée si elle est réduite et s'il existe un entier $n \geq 0$, un homomorphisme surjectif $\varphi: K\left\langle T_{1}, \ldots, T_{n}\right\rangle \rightarrow A$ tel que la norme spectrale $\|\cdot\|^{\text {sp }}$ sur $A$ soit la norme induite par $\varphi$ (Voir [BGR, définition 2, p. 254]).

Si $W$ est ouvert affine de $\bar{X}^{c}, U=r^{-1}(W)$ est une partie affinoïde de $X$ et la réduction canonique $\bar{U}^{c}$ de $U$ s'identifie canoniquement à $W$ (Voir [Bo2, théorème 3.1 , p. 20]); on dit dans ce cas que $U$ est un ouvert affinoïde formel (ou pur) de $X$. 
Un recouvrement affinoïde admissible $\mathcal{U}=\left\{U_{i}\right\}_{i \in I}$ de $X$ est dit pur (ou formel) si pour tout $i, j \in I$ la partie $U_{i} \cap U_{j}$ est ouvert pur (ou formel) de $U_{i}$; on dit alors que $(X, \mathcal{U})$ est un espace analytique formel (les situations considérées dans la suite sont élémentaires en ce sens que le recouvrement sera fini et que $U_{i} \cap U_{j}$ sera affinoïde). On dit que le recouvrement $\mathcal{U}$ est distingué si les $K$-algèbres affinoïdes $\mathcal{O}_{X}\left(U_{i}\right)$ sont distinguées.

Soient $X$ un espace analytique, $\mathcal{U}=\left\{U_{i}\right\}_{i}$ un recouvrement formel de $X$ tel que $U_{i} \cap U_{j}$ soit un ouvert affinoïde formel de $U_{i}$ pour tout $i, j$. A l'espace formel $(X, \mathcal{U})$ on associe une réduction. Les variétés algébriques affines réduites (sur $\bar{K}$ ) $\bar{U}_{i}^{c}$ se recollent le long des $\bar{U}_{i} \cap U_{j}^{c}$ en un schéma localement de type fini réduit sur $\bar{K}$, noté $(\overline{X, \mathcal{U}})$; les applications réductions $r_{i}: U_{i} \rightarrow \bar{U}_{i}^{c}$ se recollent en une application $r: X \rightarrow(\overline{X, \mathcal{U}})$ (si $\mathcal{U}$ est fini, $(\overline{X, \mathcal{U}})$ est donc une variété algébrique réduite). On dit que $r: X \rightarrow(\overline{X, \mathcal{U}})$ est la réduction analytique de $X$ associée au recouvrement pur $\mathcal{U}$ (ou la réduction analytique de l'espace formel $(X, \mathcal{U})$ ).

1.1.2. L'analytification des variétés algébriques $[\mathrm{Fr}],[\mathrm{Fr}, \mathrm{vdP}]$. - Dans tout cet article le mot variété algébrique sur un corps $K$ signifie schéma, séparé de type fini sur $K$, par point d'une variété on entendra toujours point fermé (lorsqu'il s'agira de schéma le mot point sera utilisé sans restriction).

Dans tout ce paragraphe 1.1.2, $K$ désigne un corps valué complet. Pour analytifier une variété algébrique $\left(X, \mathcal{O}_{X}\right)$ sur $K$, on définit sur $X$ une topologie de Grothendieck $\mathcal{G}$ et un faisceau structural $\mathcal{O}_{X^{\text {an }}}$ sur $(X, \mathcal{G})$. Une partie $U$ de $X$ est dite admissible si $U=X$ ou s'il existe $Y \subset X$ un ouvert affine, $f_{1}, \ldots, f_{r} \in \mathcal{O}_{X}(Y)$ tel que la $K$-algèbre $\mathcal{O}_{X}(Y)$ soit engendrée par $f_{1}, \ldots, f_{r}$ avec $U=\left\{x \in Y ;\left|f_{i}(x)\right| \leq 1\right.$ pour $1 \leq i \leq r\}$. Soit $\varphi: K\left[T_{1}, T_{2}, \ldots, T_{r}\right] \rightarrow \mathcal{O}_{X}(Y)$ l'homomorphisme défini $\operatorname{par} \varphi\left(T_{i}\right)=f_{i}$ et $\mathfrak{A}=\operatorname{ker} \varphi$, on pose alors

$$
\mathcal{O}_{X^{\text {an }}}(U) \stackrel{\text { déf }}{=} \frac{K\left\langle T_{1}, \ldots, T_{r}\right\rangle}{\mathfrak{U} K\left\langle T_{1}, \ldots, T_{r}\right\rangle}
$$

où $K\left\langle T_{1}, T_{2}, \ldots, T_{r}\right\rangle$ est l'algèbre des séries convergentes sur le polydisque unité [Fr, vdP p. 54], on peut montrer que $\mathcal{O}_{X^{\text {an }}}(U)$ ne dépend pas de la représentation de $U$ par le choix des $f_{i}$. Si $V \subset U \subset X$ sont admissibles, l'application restriction $\mathcal{O}_{X^{\text {an }}}(U) \rightarrow \mathcal{O}_{X^{\text {an }}}(V)$ est induite par les applications restrictions du faisceau $\mathcal{O}_{X}$. Enfin on pose $\mathcal{O}_{X^{\text {an }}}(X)=$ $\lim _{U} \mathcal{O}_{X^{\text {an }}}(U)$ où la limite projective est prise sur tous les admissibles $\overleftarrow{U \neq X}$.

Soit $U$ admissible, un recouvrement $\left\{U_{i}\right\}_{i \in I}$ de $U$ est dit admissible si chaque $U_{i}$ est admissible, si pour tout $V \neq X$ admissible, $V \subset U$, il existe une partie finie $F_{V} \subset I$ telle que $V \subset \bigcup_{i \in F_{V}} U_{i}$.

$$
\text { TOME } 116-1988-\mathrm{N}^{\circ} 1
$$


Alors les admissibles et les recouvrements admissibles définissent sur $X$ une topologie de Grothendieck $\mathcal{G}$. On montre alors que $\mathcal{O}_{X^{\text {an }}}$ est un faisceau $\operatorname{sur}(X, \mathcal{G})$ et que le triplet $\left(X, \mathcal{G}, \mathcal{O}_{X^{\text {an }}}\right)$ est un espace analytique. C'est l'analytification de $X$ et on le note $X^{\text {an }}$; selon ces définitions on voit qu'un admissible $U$ de $X^{\text {an }}$ avec $U \neq X^{\text {an }}$ est affinoïde.

Soient $X$ une variété algébrique intègre, $Y \subset X$ un ouvert affine, $f_{1}, f_{2}, \ldots, f_{r} \in \mathcal{O}_{X}(Y)$ avec

$$
\begin{gathered}
\mathcal{O}_{X}(Y)=K\left[f_{1}, f_{2}, \ldots, f_{r}\right] \\
U=\left\{x \in Y ;\left|f_{i}(X)\right| \leq 1 \text { pour } 1 \leq i \leq r\right\} .
\end{gathered}
$$

Alors $\|\cdot\|_{U}$ défini sur $\mathcal{O}_{X}(Y)$ par $\|f\|_{U} \stackrel{\text { déf }}{=} \sup _{x \in U}|f(x)|$ est une norme et on a canoniquement $\mathcal{O}_{X^{\text {an }}}(Y) \simeq\left(\mathcal{O}_{X}(Y),\|\cdot\|_{U}\right)^{\wedge}$ et $\|\cdot\|_{U}$ n'est autre chose que la norme spectrale de l'algèbre affinoïde $\mathcal{O}_{X^{\text {an }}}(U)$.

1.2. Réduction des courbes algébriques associées à un corps de fonctions valué.

Définition 1. - Soient $(K,|\cdot|)$ un corps valué, $K(T)$ le corps des fractions rationnelles à une variable sur $K$. On appelle valeur absolue de Gauss sur $K(T)$ associée $a ̀|\cdot|$ et à $T$ la valeur absolue notée $|\cdot|_{g}$ et définie $\operatorname{par}\left|\sum_{i} a_{i} T^{i}\right|_{g}=\max _{i}\left|a_{i}\right|$ pour tout $a_{i} \in K$.

Proposition 1 [Ma2, proposition 3, § 2]. - Soient $(K,|\cdot|)$ un corps valué complet, $\mathcal{C}$ une courbe algébrique projective sur $K$, irréductible et non singulière, $L=\mathcal{R}(\mathcal{C})$ le corps des fonctions rationnelles sur $\mathcal{C}, \mathcal{C}^{\text {an }}$ l'analytification de $\mathcal{C}$. Soient $T \in L$ transcendant sur $K$,

$$
\begin{gathered}
Z(T)=\left\{x \in \mathcal{C} ; T \in \mathcal{O}_{\mathcal{C}, x}\right\}, \quad Z\left(T^{-1}\right)=\left\{x \in \mathcal{C} ; T^{-1} \in \mathcal{O}_{\mathcal{C}, x}\right\} \\
U(T)=\{x \in Z(T) ;|T(x)| \leq 1\} \\
U\left(T^{-1}\right)=\left\{x \in Z\left(T^{-1}\right) ;\left|T^{-1}(x)\right| \leq 1\right\} \\
R=\mathcal{O}_{\mathcal{C}}(Z(T)), \quad S=\mathcal{O}_{\mathcal{C}}\left(Z\left(T^{-1}\right)\right)
\end{gathered}
$$

et $\|\cdot\|_{U(T)}^{\mathrm{sp}}\left(\right.$ resp. $\left.\|\cdot\|_{U\left(T^{-1}\right)}^{\mathrm{sp}}\right)$ la norme définie sur $R$ (resp. S) par

$$
\begin{aligned}
\|f\|_{U(T)}^{\mathrm{sp}}= & \max _{x \in U(T)}|f(x)| \quad\left(\text { resp. }\|f\|_{U\left(T^{-1}\right)}^{\mathrm{sp}}=\max _{x \in U\left(T^{-1}\right)}|f(x)|\right), \\
& \bar{R}=\left(\overline{R,\|\cdot\|_{U(T)}^{\mathrm{sp}}}\right), \quad \bar{S}=\left(\overline{S,\|\cdot\|_{U\left(T^{-1}\right)}^{\mathrm{sp}}}\right) .
\end{aligned}
$$

Soient $\left\{|\cdot|{ }_{i}\right\}_{1 \leq i \leq s}$ les valeurs absolues sur $L$ qui prolongent la valeur absolue de Gauss $|\cdot|_{g}$ sur $K(T)$ associée à $T$ et $\grave{a}|\cdot|,\|\cdot\|$ la norme sur $L$ définie par $\|f\|=\max _{1 \leq i \leq s}|f|_{i}$. Alors on a les résultats qui suivent. 
1) Soient $f \in R$ (resp. $S$ ), $a_{0}(T)+a_{1}(T) X+\cdots+X^{r}$ (resp. $\left.a_{0}\left(T^{-1}\right)+a_{1}\left(T^{-1}\right) X+\cdots+X^{r}\right)$ le polynôme irréductible de $f$ sur $K(T)$, donc sur $K[T]$ (resp. $K\left[T^{-1}\right]$ ), alors

$$
\max _{0 \leq j<r}\left|a_{j}(T)\right|_{g}^{1 / r-j}=\|f\|_{U(T)}^{\mathrm{sp}}
$$

resp.

$$
\max _{0 \leq j<r}\left|a_{j}\left(T^{-1}\right)\right|_{g}^{1 / r-j}=\|f\|_{U\left(T^{-1}\right)}^{\mathrm{sp}} \text {. }
$$

2) On a $\bar{L}=\operatorname{Fr}(\bar{R})=\operatorname{Fr}(\bar{S})=\bar{L}^{1} \times \bar{L}^{2} \times \cdots \times \bar{L}^{s}$ où $\bar{L}^{i}=\left(\overline{L,|\cdot|_{i}}\right)$, $\bar{L}=(\overline{L,\|\cdot\|})$ où $\operatorname{Fr}(\bar{R})$ désigne l'anneau total des fractions de $\bar{R}$. (resp. $\operatorname{Fr}(\bar{S})$ désigne l'anneau total des fractions de $\bar{S})$. de $\mathcal{C}^{\text {an }}$,

3) Les parties $U(T), U\left(T^{-1}\right)$ sont des parties affinoïdes admissibles

$$
\begin{aligned}
\mathcal{O}_{\mathcal{C}^{\text {an }}}(U(T))=\left(R,\|\cdot\|_{U(T)}^{\mathrm{sp}}\right)^{\wedge}, & \mathcal{O}_{\mathcal{C}^{\text {an }}}\left(U\left(T^{-1}\right)\right)=\left(S,\|\cdot\|_{U\left(T^{-1}\right)}^{\mathrm{sp}}\right)^{\wedge} \\
\overline{\mathcal{O}_{\mathcal{C}^{\mathrm{an}}}(U(T))}=\bar{R}, & \overline{\mathcal{O}_{\mathcal{C}^{\mathrm{an}}}\left(U\left(T^{-1}\right)\right)}=\bar{S} .
\end{aligned}
$$

4) L'ensemble $\mathcal{U}=\left\{U(T), U\left(T^{-1}\right)\right\}$ est un recouvrement pur de $\mathcal{C}^{\text {an }}$ et la réduction $r: \mathcal{C}^{\text {an }} \rightarrow\left(\overline{\mathcal{C}^{\text {an }}, \mathcal{U}}\right)=\mathcal{E}$ est une courbe algébrique, projective, réduite, connexe. On a $r\left(U(T) \cap U\left(T^{-1}\right)\right)$ qui est dense dans $\mathcal{E}$ et $\mathcal{R}(\mathcal{E})=\bar{L}=\bar{L}^{1} \times \bar{L}^{2} \times \cdots \times \bar{L}^{s} .(\mathcal{R}(\mathcal{E})$ est l'anneau des fonctions rationnelles sur $\mathcal{E}$, cf. Définition $3, \S 3)$.

5) Soient $1 \leq i \leq s, \mathcal{E}_{i}$ l'unique composante irréductible de $\mathcal{E}$ telle que $\bar{L}^{i}=\mathcal{R}\left(\mathcal{E}_{i}\right), W_{i}=D\left(\bar{f}_{i}\right) \subset r(U(T)) \cap \mathcal{E}_{i}$ un ouvert principal non vide de $r(U(T)),\|\cdot\|_{r^{-1}\left(W_{i}\right)}^{\mathrm{sp}}$ la norme spectrale sur l'ouvert affinö̈de admissible $r^{-1}\left(W_{i}\right)$. Alors on a pour tout $f \in R,|f|_{i}=\|f\|_{r^{-1}\left(W_{i}\right)}^{\mathrm{sp}}$, ainsi $\|\cdot\|_{r^{-1}\left(W_{i}\right)}^{\mathrm{sp}}$ induit sur $L \subset \operatorname{Fr}\left(\mathcal{O}_{\mathcal{C}^{\text {an }}}\left(r^{-1}\left(W_{i}\right)\right)\right)$ une unique valeur absolue, c'est $|\cdot|_{i}$ (on a donc une bijection explicite entre les valeurs absolues $\left\{|\cdot|_{i}\right\}_{1 \leq i \leq s}$ et les composantes irréductibles de $\mathcal{E}$ (resp. les composantes irréductibles de $r(U(T)))$.

\subsection{Le corps $L \widehat{K}$ et ses valeurs absolues.}

Définition 2. - Soient $(K,|\cdot|)$ un corps valué, $\widehat{K}$ le complété de $K$, $L / K$ un corps de fonctions à une variable dont le corps des constantes est purement inséparable sur $K$. Il s'ensuit que l'idéal $\mathfrak{N}$ des nilpotents de la $K$-algèbre $\widehat{K} \otimes_{K} L$ est un idéal premier [Bki, $\S 17.2$, corollaire, p. 134], ainsi $M \stackrel{\text { déf }}{=} \operatorname{Fr}\left(\widehat{K} \otimes_{K} L / \mathfrak{N}\right)$ est un corps (Fr $A$ désigne l'anneau total des fractions). Les corps $\widehat{K}$ et $L$ s'injectent canoniquement dans $M$ en des

$$
\text { TOME } 116-1988-\mathrm{N}^{\mathrm{O}} 1
$$


corps toujours notés $\widehat{K}$ et $L$; ainsi $M$ est le compositum des corps $\widehat{K}$ et $L$, c'est pourquoi on note $M$ par $L \widehat{K}$.

Proposition 2. - Soient $(K,|\cdot|)$ un corps valué, $\widehat{K}$ le complété de $K, L / K$ un corps de fonctions à une variable dont le corps des constantes est purement inséparable sur $K, \mathcal{W}$ (resp. $\mathcal{W}^{\wedge}$ ) l'ensemble des valeurs absolues $|\cdot|^{\prime}$ sur $L$ (resp. L $\widehat{K}$ ) qui prolongent $|\cdot|$ et telles que $\left(\overline{L,|\cdot|^{\prime}}\right)$ (resp. $\left(\overline{L \widehat{K},|\cdot|^{\prime}}\right)$ soit un corps de fonctions à une variable sur $(\overline{K,|\cdot|})$ (resp. $(\widehat{\widehat{K},|\cdot|)})$. Alors l'application $|\cdot|^{\prime} \mapsto|\cdot|_{L}^{\prime}$ de $\mathcal{W}^{\wedge}$ dans $\mathcal{W}$ est une bijection $\left(|\cdot|_{L}^{\prime}\right.$ désigne la restriction de $|\cdot|^{\prime}$ à $\left.L\right)$.

Démonstration. - Montrons que l'application est injective. Soient $|\cdot|_{1}$, $|\cdot|_{2} \in \mathcal{W}^{\wedge}$ avec $|\cdot|_{1 L}=|\cdot|_{2 L}, x=\sum_{i} a_{i} b_{i} \in L \widehat{K}, a_{i} \in \widehat{K}, b_{i} \in L, x \neq 0$. Il existe $a_{i}^{\prime} \in K$ avec $\left|a_{i}^{\prime}-a_{i}\right|\left|b_{i}\right|_{1}<\inf \left(|x|_{1},|x|_{2}\right)$. On a

$$
x=\sum_{i} a_{i} b_{i}=\sum_{i} a_{i}^{\prime} b_{i}+\sum_{i}\left(a_{i}-a_{i}^{\prime}\right) b_{i} .
$$

Ainsi $|x|_{j}=\left|\sum_{i} a_{i}^{\prime} b_{i}\right|_{j}$ pour $j=1,2$. Comme $a_{i}^{\prime} \in K$ on a $\left|\sum_{i} a_{i}^{\prime} b_{i}\right|_{1}=$ $\left|\sum_{i} a_{i}^{\prime} b_{i}\right|_{2}$, ainsi $|x|_{1}=|x|_{2}$. Ce qui montre que l'application est injective.

Montrons que l'application est surjective. Soient $|\cdot| \in \mathcal{W}$ et $T \in L$ tels que $|T|^{\prime}=1$ et que $T$ soit résiduellement transcendant sur $K$. Soit $\widehat{L}=\left(L,|\cdot|^{\prime}\right)^{\wedge}$, l'injection de $K$ dans $L$ se prolonge par continuité en une injection de $\widehat{K}$ dans $\widehat{L}$; ainsi il existe un homomorphisme $\psi: \widehat{K} \otimes_{K} L \rightarrow$ $\widehat{L}$. De plus $T \in L$ est transcendant sur $\widehat{K}$, puisque résiduellement transcendant, ainsi $\widehat{K}$ et $L$ sont algébriquement disjoints sur $K$ et $\operatorname{ker} \psi=$ $\mathfrak{N}$ [Bki, $\S 17.2$, proposition 1, p. 132]. L'homomorphisme $\psi$ induit une injection $\rho: M=\operatorname{Fr}\left(\widehat{K} \otimes_{K} L / \mathfrak{N}\right) \rightarrow \widehat{L}$. Par suite le prolongement canonique de $|\cdot|^{\prime}$ à $\widehat{L}$ induit (via $\rho$ ) une valeur absolue $|\cdot|^{\prime \prime}$ sur $M=L \widehat{K}$ telle que $|\cdot|_{L}^{\prime \prime}=|\cdot|^{\prime}$.

\section{Prolongement de la valeur absolue de Gauss et réduction analytique}

Le but de ce paragraphe, le THÉORÈme 1 , est de montrer qu'une valeur absolue $|\cdot|$ sur un corps de fonction $L / K$ peut être considérée comme l'unique prolongement d'une valeur absolue de Gauss sur une extension transcendante pure de $K$ si et seulement si une propriété d'une certaine réduction analytique de la courbe $\mathcal{C}_{L}$ est satisfaite $\left(\mathcal{C}_{L}\right.$ est l'unique courbe sur $K$, projective, non singulière dont $L$ est le corps des fonctions rationnelles).

Pour faire cela nous avons besoin de deux résultats préliminaires, le Lemme 1 et la Proposition 3. 
LEMME 1. - Soient $k$ un corps, $A$ une $k$-algèbre de type fini, de dimension 1 , intègre, $B$ la normalisation de $A$ dans $\operatorname{Fr}(A),\left\{\mathfrak{M}_{1}, \mathfrak{M}_{2}, \ldots, \mathfrak{M}_{s}\right\}$ l'ensemble des idéaux maximaux de $B$ tels que $A_{A \cap m_{i}}$ ne soit pas normal, $f \in \mathfrak{M}_{1} \cap \mathfrak{M}_{2} \ldots \cap \mathfrak{M}_{s}$. Alors il existe un entier $N>0$ tel que $f^{N} \in A$.

Proposition 3. - Soient $K$ un corps valué complet, $X$ une variété algébrique sur $K$, projective, réduite, pure de dimension $1, X^{\text {an }}$ l'analytification de $X, \mathcal{U}$ un recouvrement distingué pur, fini et $r: X^{\text {an }} \rightarrow \mathcal{E}$, la réduction analytique associée. Soient $z_{1}, z_{2}, \ldots, z_{\tau} \in X$ tels que $r\left(z_{i}\right)$ soit régulier pour $1 \leq i \leq \tau$, que $\mathcal{E}-\left\{r\left(z_{1}\right), \ldots, r\left(z_{\tau}\right)\right\}$ soit affine et que $r\left(z_{i}\right) \neq r\left(z_{i^{\prime}}\right)$ pour $i \neq i^{\prime}$. Alors il existe $f \in \mathcal{O}_{X}\left(X-\left\{z_{1}, \ldots, z_{\tau}\right\}\right)$ tel que $f \notin \mathcal{O}_{X, z_{i}}$ pour $1 \leq i \leq \tau$ et que

$$
r^{-1}\left(\mathcal{E}-\left\{r\left(z_{1}\right), \ldots, r\left(z_{\tau}\right)\right)=\left\{z \in X ; f \in \mathcal{O}_{X, z} \text { et }|f(z)| \leq 1\right\} .\right.
$$

Démonstration. - Voir [Pz, Proposition 3, p. 21].

THÉORÈme 1. - Soient $L / K$ un corps de fonctions d'une variable dont le corps des constantes est purement inséparable sur $K,|\cdot|$ une valeur absolue sur $L$ telle que $\bar{L} / \bar{K}$ soit un corps de fonctions d'une variable où $\bar{L}$ (resp. $\bar{K}$ ) désigne le corps résiduel de $L$ (resp. K); notons $|\cdot|_{0}$ la restriction de $|\cdot| \grave{a} K$. Alors les propriétés suivantes sont équivalentes.

i) Il existe $T \in L$ transcendant sur $K$ tel que $|\cdot|$ soit l'unique prolongement à $L$ de la valeur absolue de Gauss sur $K(T)$ associée à |. lo et à $T$.

ii) Il existe $Z \in L \widehat{K}$ transcendant sur $\widehat{K}$ tel que $|\cdot|$ soit un prolongement à $L \widehat{K}$ de la valeur absolue de Gauss sur $\widehat{K}(Z)$ associée $\grave{a} Z$ et $\grave{a}$ | $\left.\right|_{0}$ et qui possède la propriété qui suit. Soient $r: \mathcal{C} \widehat{\mathcal{K}}_{K} \rightarrow \mathcal{E}$ la réduction analytique associée à $Z, \mathcal{E}_{1}$ la composante irréductible de $\mathcal{E}$ correspondant $\grave{a}|\cdot|$; alors pour tout ouvert non vide $W \subset \mathcal{E}_{1}$, il existe $x_{W} \in \mathcal{C}_{L}$ tel que $r(y) \in W$, pour tout point $y \in \mathcal{C}_{L \widehat{K}}$ au-dessus de $x_{W}$.

iii) Soit $Z^{\prime} \in L \widehat{K}$ transcendant sur $\widehat{K}$ tel que $|\cdot|$ soit un prolongement à $L \widehat{K}$ de la valeur absolue de Gauss sur $\widehat{K}\left(Z^{\prime}\right)$ associée $\grave{a}|\cdot|_{0}$ et $\grave{a}$ $Z^{\prime}$. Soient $r^{\prime}: \mathcal{C}_{L \widehat{K}} \rightarrow \mathcal{E}^{\prime}$ la réduction analytique associée à $Z^{\prime}$, $\mathcal{E}_{1}^{\prime}$ la composante irréductible de $\mathcal{E}^{\prime}$ correspondant $\grave{a}|\cdot|$; alors pour tout ouvert non vide $V \subset \mathcal{E}_{1}^{\prime}$, il existe $x_{V} \in \mathcal{C}_{L}$ tel que $r^{\prime}(y) \in V$ pour tout point $y \in \mathcal{C}_{L \widehat{K}}$ au-dessus de $x_{V}$.

Démonstration.

i) implique ii). Soient $T \in L$ satisfaisant i),

$$
\rho: \mathcal{C}_{L} \rightarrow \mathbf{P}_{K}^{1} \quad\left(\text { resp. } \widehat{\rho}: \mathcal{C}_{L \widehat{K}} \rightarrow \mathbf{P}_{\widehat{K}}^{1}\right)
$$

TOME $116-1988-\mathrm{N}^{\circ} 1$ 
le morphisme défini par l'inclusion $K(T) \subset L$ (resp. $\widehat{K}(T) \subset L \widehat{K})$, soient

$$
\begin{aligned}
U_{1}(T) & =\left\{x \in \mathbf{P}_{\widehat{K}}^{1} ; T \in \mathcal{O}_{\mathbf{P}_{\widehat{K}}^{1}, x} \text { et }|T(x)| \leq 1\right\}, \\
U_{1}\left(T^{-1}\right) & =\left\{x \in \mathbf{P}_{\widehat{K}}^{1} ; T^{-1} \in \mathcal{O}_{\mathbf{P}_{\widehat{K}}^{1}, x} \text { et }\left|T^{-1}(x)\right| \leq 1\right\}, \\
U(T) & =\left\{x \in C_{L \widehat{K}} ; T \in \mathcal{O}_{C_{L \widehat{K}}, x} \text { et }|T(x)| \leq 1\right\}, \\
U\left(T^{-1}\right) & =\left\{x \in C_{L \widehat{K}} ; T^{-1} \in \mathcal{O}_{C_{L \widehat{K}}, x} \text { et }\left|T^{-1}(x)\right| \leq 1\right\} .
\end{aligned}
$$

Alors $\mathcal{U}_{1}=\left\{U_{1}(T), U_{1}\left(T^{-1}\right)\right\}$ (resp. $\mathcal{U}=\left\{U(T), U\left(T^{-1}\right)\right\}$ ) est un recouvrement pur de $\mathbf{P}_{\widehat{K}}^{1}\left(\right.$ resp. $\left.C_{L \widehat{K}}\right)$. On a

$$
U(T)=\widehat{\rho}^{-1}\left(U_{1}(T)\right) \quad\left(\text { resp. } U\left(T^{-1}\right)=\widehat{\rho}^{-1}\left(U_{1}\left(T^{-1}\right)\right)\right) .
$$

Soient $r_{1}: \mathbf{P}_{\widehat{K}}^{1} \rightarrow \mathbf{P}_{\bar{K}}^{1}$ la réduction définie par $\mathcal{U}_{1}, r: \mathcal{C}_{L \widehat{K}}^{\text {an }} \rightarrow \mathcal{E}$ celle définie par $\mathcal{U}$, il suit que $\hat{\rho}$ induit un morphisme surjectif et fini $\bar{\rho}: \mathcal{E} \rightarrow \mathbf{P} \frac{1}{K}$ avec $\bar{\rho}_{\circ} r=r_{1 \circ} \widehat{\rho}$.

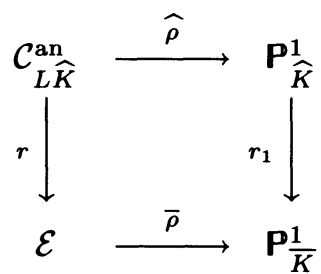

Comme $|\cdot|$ est l'unique valeur absolue prolongeant la valeur absolue de Gauss sur $\widehat{K}(T)$ associée à $|\cdot|_{0}$ et à $T(\S 1.3$, Proposition 2), on a $\mathcal{E}=\mathcal{E}_{1}$. Soit $W$ un ouvert de $\mathcal{E}$, non vide, il existe donc un ouvert non vide $V$ de $\mathbf{P}_{\bar{K}}^{1}$ tel que $\bar{\rho}^{-1}(V) \subset W$. Comme $V$ est dense dans $\mathbf{P} \frac{1}{K}$ il existe $\xi \in V \cap \operatorname{Spm}(\bar{K}[T])$, on a $\mathfrak{m}_{\xi}=p(T) \bar{K}[T]$ où $p(T)=$ $p_{0}+p_{1} T+\cdots+p_{m-1} T^{m-1}+T^{m}$ est un polynôme unitaire et irréductible de $\bar{K}[T]$. Soit $P(T)=a_{0}+a_{1} T+\cdots+a_{m-1} T^{m-1}+T^{m} \in K^{\circ}[T]$ avec $\bar{a}_{i}=p_{i}$, où $K^{\circ}=\{a \in K$ tel que $|a| \leq 1\}$. Clairement $P(T)$ est un polynôme irréductible de $\widehat{K}[T]$ (donc aussi de $K[T]$ ). Soit $x \in \mathcal{C}_{L}$ tel que $\rho(x)$ soit le point de $\mathbf{P}_{\widehat{K}}^{1}$ correspondant au maximal $P(T) K[T]$ de $K[T]$.

Comme $P(T) \widehat{K}[T]$ est maximal, pour tout $y \in \mathcal{C}_{L \widehat{K}}$ au-dessus de $x$ le point $\widehat{\rho}(y)$ correspond au maximal $P(T) \widehat{K}[T]$ de $\widehat{K}[T]$. Il suit de cela que $r_{1}(\hat{\rho}(y))=\xi$ et comme $r_{1 \circ} \widehat{\rho}=\bar{\rho}_{\circ} r$, on a $\bar{\rho}(r(y))=\xi$, ce qui montre que $r(y) \in \bar{\rho}^{-1}(V) \subset W$.

ii) implique iii). Soient $Z \in L \widehat{K}$ satisfaisant ii), $Z^{\prime} \in L \widehat{K}$ satisfaisant les hypothèses de iii),

$$
\begin{gathered}
\mathcal{U}=\left\{U(Z), U\left(Z^{-1}\right)\right\}, \quad \mathcal{U}^{\prime}=\left\{U\left(Z^{\prime}\right), U\left(Z^{\prime-1}\right)\right\} \\
\mathcal{V}=\left\{U(Z) \cap U\left(Z^{\prime}\right), U(Z) \cap U\left(Z^{\prime-1}\right), U\left(Z^{-1}\right) \cap U\left(Z^{\prime}\right), U\left(Z^{-1}\right) \cap U\left(Z^{\prime-1}\right)\right\} .
\end{gathered}
$$


Ainsi $\mathcal{U}, \mathcal{U}^{\prime}, \mathcal{V}$ sont des recouvrements purs de $\mathcal{C}_{L \widehat{K}}^{\text {an }}$. Soient

$$
r: \mathcal{C}_{L \widehat{K}}^{\mathrm{an}} \rightarrow \mathcal{E}, \quad r^{\prime}: \mathcal{C}_{L \widehat{K}}^{\mathrm{an}} \rightarrow \mathcal{E}^{\prime} \quad \text { et } \quad r^{\prime \prime}: \mathcal{C}_{L \widehat{K}}^{\mathrm{an}} \rightarrow \mathcal{E}^{\prime \prime}
$$

les réductions analytiques associées; comme $\mathcal{V}$ est plus fin que $\mathcal{U}$ (resp. $\mathcal{U}^{\prime}$ ), il existe un morphisme $\varphi: \mathcal{E}^{\prime \prime} \rightarrow \mathcal{E}\left(\right.$ resp. $\varphi^{\prime}: \mathcal{E}^{\prime \prime} \rightarrow \mathcal{E}^{\prime}$ ) tel que $r=\varphi_{\circ} r^{\prime \prime}\left(\right.$ resp. $\left.r^{\prime}=\varphi_{\circ}^{\prime} r^{\prime \prime}\right)$.

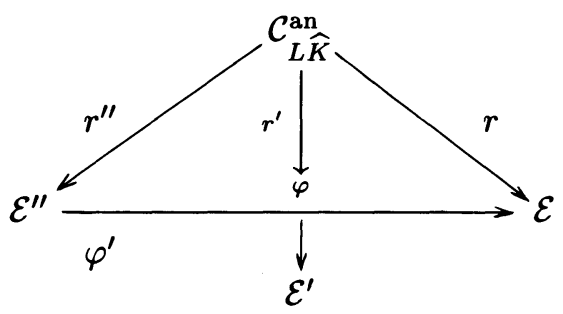

Soient $\mathcal{E}_{1}^{\prime \prime}, \mathcal{E}_{2}^{\prime \prime}, \ldots, \mathcal{E}_{s}^{\prime \prime}$ les composantes irréductibles de $\mathcal{E}^{\prime \prime}$ telles que $\operatorname{card}\left(\varphi\left(\mathcal{E}_{i}^{\prime \prime}\right)\right)=1$. Soit $\mathcal{E}_{1}$ la composante de $\mathcal{E}$ correspondant à $|\cdot|$, il existe un ouvert non vide $W$ de $\mathcal{E}$ avec $W \subset \mathcal{E}_{1}$ et $W \cap \varphi\left(\mathcal{E}_{1}^{\prime \prime} \cup \mathcal{E}_{2}^{\prime \prime} \ldots \cup \mathcal{E}_{s}^{\prime \prime}\right)=\emptyset$. Alors $W$ est affine (si $s=0$ on peut toujours choisir $W$ affine), montrons que $\varphi^{-1}(W)$ est affine; soit $\mathcal{E}_{j}^{\prime \prime}$ une composante irréductible de $\mathcal{E}^{\prime \prime}$, on a $\varphi^{-1}(W) \cap \mathcal{E}_{j}^{\prime \prime} \neq \mathcal{E}_{j}^{\prime \prime}$, sinon $\varphi\left(\mathcal{E}_{j}^{\prime \prime}\right)$ serait un point parce que $\mathcal{E}_{j}^{\prime \prime}$ est projectif puisque $\mathcal{E}^{\prime \prime}$ l'est [Fr, Ma, $\S 1.4$, lemme 3] et que $W$ est affine, or le choix de $W$ montre que c'est impossible. Ensuite

$$
r: r^{-1}(W) \rightarrow W\left(\text { resp. } r^{\prime \prime}: r^{\prime \prime-1}\left(\varphi^{-1}(W)\right) \rightarrow \varphi^{-1}(W)\right)
$$

coïncide avec la réduction canonique, ce qui montre que $\varphi: \varphi^{-1}(W) \rightarrow W$ est un isomorphisme.

Soit $\mathcal{E}_{1}^{\prime}$ la composante irréductible de $\mathcal{E}^{\prime}$ correspondant à $|\cdot|$, pour les mêmes raisons il existe un ouvert non vide $W^{\prime}$ de $\mathcal{E}^{\prime}$ avec $W^{\prime} \subset \mathcal{E}_{1}^{\prime}$ tel que $\varphi^{\prime}: \varphi^{\prime-1}\left(W^{\prime}\right) \rightarrow W^{\prime}$ soit un isomorphisme.

Montrons que

$$
\varphi^{-1}(W) \cap \varphi^{\prime-1}\left(W^{\prime}\right) \neq \emptyset .
$$

Supposons $\varphi^{-1}(W) \cap \varphi^{\prime-1}\left(W^{\prime}\right)=\emptyset$. Il s'ensuit que $\varphi^{-1}(W) \cup \varphi^{\prime-1}\left(W^{\prime}\right)$ est ouvert affine de $\mathcal{E}^{\prime \prime}$, que

$$
r^{\prime \prime-1}\left(\varphi^{-1}(W)\right) \cup r^{\prime \prime-1}\left(\varphi^{\prime-1}\left(W^{\prime}\right)\right)
$$

est un admissible affinoïde de $\mathcal{C}_{L \widehat{K}}^{\text {an }}$, et on a

$$
r^{\prime \prime-1}\left(\varphi^{-1}(W)\right) \cap r^{\prime \prime-1}\left(\varphi^{\prime-1}\left(W^{\prime}\right)\right)=\emptyset
$$

TOME $116-1988-\mathrm{N}^{\circ} 1$ 
avec $r^{\prime \prime-1}\left(\varphi^{-1}(W)\right)$ et $r^{\prime \prime-1}\left(\varphi^{\prime-1}\left(W^{\prime}\right)\right)$ affinoïdes. Par suite il existe $f \in L \widehat{K}$ avec

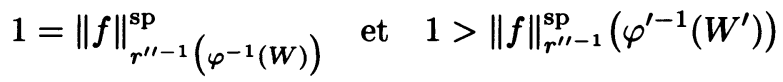

[Fr, Ma, Théorème 4, $\S 2.4]$. Comme $r^{\prime \prime-1}\left(\varphi^{-1}(W)\right)=r^{-1}(W)$ (resp. $\left.r^{\prime \prime-1}\left(\varphi^{\prime-1}\left(W^{\prime}\right)\right)=r^{\prime-1}\left(W^{\prime}\right)\right)$, on a $|f|=\|f\|_{r(W)}^{\mathrm{sp}}\left(\right.$ resp. $\left.|f|=\|f\|_{r^{\prime}\left(W^{\prime}\right)}^{\mathrm{sp}}\right)$ (Proposition 1, $\S 1.2$ ) ce qui est une contradiction.

Soit $V=\varphi^{-1}(W) \cap \varphi^{\prime-1}\left(W^{\prime}\right)$, il suit que $\varphi(V)$ (resp. $\left.\varphi^{\prime}\left(V^{\prime}\right)\right)$ est un ouvert de $\mathcal{E}$ (resp. $\mathcal{E}^{\prime}$ ), que $V=\varphi^{-1}(\varphi(V))$ (resp. $\left.V=\varphi^{\prime-1}\left(\varphi^{\prime}(V)\right)\right)$, que $\varphi: V \rightarrow \varphi(V)$ (resp. $\varphi^{\prime}: V \rightarrow \varphi^{\prime}(V)$ ) est un isomorphisme.

Soit $W_{1}^{\prime}$ un ouvert de $\mathcal{E}^{\prime}$ avec $W_{1}^{\prime} \subset \mathcal{E}_{1}^{\prime}$, ainsi $W_{1} \stackrel{\text { déf }}{=} \varphi\left(\varphi^{\prime-1}\left(\varphi^{\prime}(U) \cap W_{1}^{\prime}\right)\right)$ est un ouvert de $\mathcal{E}$ avec $W_{1} \subset \mathcal{E}_{1}$. Par ii) il existe $x \in \mathcal{C}_{L}$ tel que pour tout $y \in \mathcal{C}_{L \widehat{K}}$ au-dessus de $x$ on ait $r(y) \in W_{1}$. Il suit facilement de ce qui précède que $r^{\prime}(y) \in W_{1}^{\prime}$ pour tout $y$ au-dessus de $x$. Ce qui montre iii).

iii) implique i). Si $L^{\prime} / K^{\prime}$ est un corps de fonctions d'une variable, on note $\mathcal{C}_{L^{\prime}}$ l'unique courbe sur $K^{\prime}$, projective non singulière telle que $\mathcal{R}\left(\mathcal{C}_{L^{\prime}}\right)=L^{\prime}$, i.e. telle que l'anneau des fonctions rationnelles sur $\mathcal{C}_{L^{\prime}}$ soit le corps $L^{\prime}$.

Par le théorème $1, \S 3$ de [Ma2], il existe $T \in L \widehat{K}$ transcendant sur $\widehat{K}$ tel que $|\cdot|$ soit l'unique prolongement à $L \widehat{K}$ de la valeur absolue de Gauss sur $\widehat{K}(T)$ associée à $|\cdot|_{0}$ et $T$. Soient $\mathcal{C}_{L \widehat{K}}$ la courbe associée à $L \widehat{K} / \widehat{K}$, $\mathcal{U}=\left\{U(T), U\left(T^{-1}\right)\right\}$ le recouvrement pur de $\mathcal{C}_{L \widehat{K}}^{\text {an }}$ défini par

$$
\begin{gathered}
U(T)=\left\{z \in \mathcal{C}_{L \widehat{K}} ; T \in \mathcal{O}_{\mathcal{C}_{L \widehat{K}}, z} \text { et }|T(z)| \leq 1\right\} \\
U\left(T^{-1}\right)=\left\{z \in \mathcal{C}_{L \widehat{K}} ; T^{-1} \in \mathcal{O}_{\mathcal{C}_{L \widehat{K}}, z} \text { et }\left|T^{-1}(z)\right| \leq 1\right\}
\end{gathered}
$$

Soit $r: \mathcal{C}_{L \widehat{K}}^{\text {an }} \rightarrow \mathcal{E}$ la réduction analytique associée à $\mathcal{U}$, alors $\mathcal{E}$ est irréductible (Proposition 1, § 1.3).

$\alpha)$ Il s'agit de démontrer qu'il existe un corps $K_{2}$ normal fini sur $\widehat{K}$ tel que le recouvrement pur de $\mathcal{C}_{L K_{2}}$ associé à $T$ (selon le $\S 1.2$, Proposition 1$)$, soit distingué $\left(L K_{2}\right.$ est le compositum pris dans $(L \widehat{K})^{\text {alg }}$ = clôture algébrique de $L \widehat{K})$.

Soient $\mathcal{C}_{L}$ la courbe associée à $L / K,\left(\mathcal{C}_{L}\right)_{(\widehat{K})}$ la variété obtenue par extension des scalaires à $\widehat{K}$ et $Z=\left(\left(\mathcal{C}_{L}\right)_{(\widehat{K})}\right)_{\text {red }}$ la variété réduite canoniquement associée à $\left(\mathcal{C}_{L}\right)_{(\widehat{K})}$; ainsi $\mathcal{C}_{L \widehat{K}}$ est la normalisation de $Z$, soit $\rho: \mathcal{C}_{L \widehat{K}} \rightarrow Z$ le morphisme de normalisation. 
$\left.\alpha_{1}\right)$ Soit $E$ la clôture algébrique de $\widehat{K}$ dans $(L \widehat{K})^{\text {alg }}$. Il existe $K_{1}^{\prime}$ fini sur $\widehat{K}$ tel que $L K_{1}^{\prime} / K_{1}^{\prime}$ soit séparable, que $K_{1}^{\prime}$ soit algébriquement fermé dans $L K_{1}^{\prime}$ et tel que $\left[L K_{1}^{\prime}: K_{1}^{\prime}(T)\right]=[L E: E(T)]=n$. Il en résulte que, pour tout corps intermédiaire $K_{1}^{\prime} \subset M \subset E$, on a $[L M: M(T)]=n$. D'autre part, il suit que $\mathcal{C}_{L \widehat{K}_{1}^{\prime}}$ est géométriquement intègre [Bki, corollaire, $\S 17, \mathrm{n}^{\circ} 2$, p. 134].

$\alpha_{2}$ ) Soit $A$ (resp. $B$ ) la clôture intégrale de $E[T]$ (resp. $E\left[T^{-1}\right]$ ) dans $L E$. Il existe $a_{1}, \ldots, a_{n} \in A$ (resp. $b_{1}, \ldots, b_{n} \in B$ ) avec $A=\bigoplus_{i=1}^{n} E[T] a_{i}$ (resp. $B=\bigoplus_{i=1}^{n} E\left[T^{-1}\right] b_{i}$ ). On peut écrire $a_{i}=\sum_{j} \lambda_{i j} u_{i j}$ avec $\lambda_{i j} \in L$, $u_{i j} \in E$ (resp. $b_{i}=\sum_{j} \mu_{i j} v_{i j}, \mu_{i j} \in L, v_{i j} \in E$ ). Soit alors $K_{1}$ la clôture normale de $K_{1}^{\prime}\left[u_{i j}, v_{i j}\right]_{i, j}$ dans $L E$. On a $a_{i}, b_{i} \in L K_{1}$ pour $1 \leq i \leq n$. Soit $K^{\prime}$ un corps avec $K_{1} \subset K^{\prime} \subset E$, alors on a

$$
\begin{array}{ll} 
& A \cap L K^{\prime}=\bigoplus_{i} K^{\prime}[T] a_{i}=K^{\prime} \otimes_{K_{1}}\left(\bigoplus_{i} K_{1}[T] a_{i}\right) \\
\text { resp. } & B \cap L K^{\prime}=\bigoplus_{i} K^{\prime}\left[T^{-1}\right] b_{i}=K^{\prime} \otimes_{K_{1}}\left(\bigoplus_{i} K_{1}\left[T^{-1}\right] b_{i}\right)
\end{array}
$$

et, d'autre part, $A \cap L K^{\prime}$ (resp. $B \cap L K^{\prime}$ ) est la clôture intégrale de $K^{\prime}[T]$ (resp. $K^{\prime}\left[T^{-1}\right]$ ) dans $L K^{\prime}$, ce qui montre que

$$
\mathcal{C}_{L K^{\prime}}=\left(\mathcal{C}_{L K_{1}}\right)_{\left(K^{\prime}\right)}
$$

$\operatorname{car} \mathcal{C}_{L K^{\prime}}$ est la normalisation de $\left(\left(\mathcal{C}_{L K_{1}}\right)_{\left(K^{\prime}\right)}\right)_{\text {red }}=\left(\mathcal{C}_{L K_{1}}\right)_{\left(K^{\prime}\right)}$.

On a alors $\mathcal{C}_{L K^{\prime}}^{\text {an }}=\left(\mathcal{C}_{L K_{1}}^{\text {an }}\right)_{\left(K^{\prime}\right)}$; en plus $\mathcal{C}_{L K_{1}}$ géométriquement réduit implique $\mathcal{C}_{L K_{1}}^{\mathrm{an}}$ géométriquement réduit [Fr, exercice 20, p. 383].

$\left.\alpha_{3}\right)$ On rappelle le résultat suivant [Bo1, lemma 2.7, p. 5] ou encore [Fr, exercice 12, p. 375] :

Soit $k$ un corps valué complet, $\Omega$ le complété de la clôture algébrique de $k, \mathcal{A}$ une $k$-algèbre affinoïde telle que $\mathcal{A} \widehat{\otimes}_{k} \Omega$ soit réduite. Alors il existe un corps $k_{0} \supset k$ fini et séparable sur $k$ tel que la $k_{0}^{\prime}$-algèbre $\mathcal{A}_{0}=\mathcal{A} \widehat{\otimes}_{k} k_{0}^{\prime}$ soit distinguée pour tout corps valué complet $k_{0}^{\prime}$ contenant $k_{0}$.

Si l'on applique ce résultat à

$$
\mathcal{A}=\mathcal{O}_{\mathcal{C}_{L K_{1}}^{\text {an }}}\left(U_{1}(T)\right) \quad\left(\operatorname{resp} . \mathcal{O}_{\mathcal{C}_{L K_{1}}^{\text {an }}}\left(U_{1}\left(T^{-1}\right)\right)\right)
$$

où $\mathcal{U}_{1}=\left\{U_{1}(T), U_{1}\left(T^{-1}\right)\right\}$ est le recouvrement pur de $\mathcal{C}_{L K_{1}}^{\text {an }}$ associé à $T$, on voit qu'il suffit de prendre pour $K_{2}$ la clôture normale de $k_{0}^{\prime}$ sur $\widehat{K}$.

$\beta$ ) Soit $\mathcal{U}_{2}=\left\{U_{2}(T), U_{2}\left(T^{-1}\right)\right\}$ le recouvrement pur de $\mathcal{C}_{L K_{2}}^{\text {an }}$ associé à $T$, il est donc distingué $(\operatorname{par} \alpha))$. Soit $r_{2}: \mathcal{C}_{L K_{2}}^{\text {an }} \rightarrow \mathcal{E}_{2}$ la réduction

TOME $116-1988-\mathrm{N}^{\circ} 1$ 
analytique associée à $\mathcal{U}_{2}, \varphi: \mathcal{C}_{L K_{2}} \rightarrow \mathcal{C}_{L \widehat{K}}$ le morphisme (sur $\widehat{K}$ ) fini induit par l'inclusion $L \widehat{K} \subset L K_{2}$.

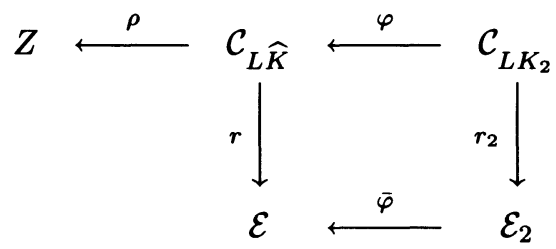

$\varphi$ induit un morphisme fini $\bar{\varphi}: \mathcal{E}_{2} \rightarrow \mathcal{E}$ tel que $r_{0} \varphi=\bar{\varphi}_{0} r_{2}$.

Soit $W_{2}=\left(\mathcal{E}_{2}\right)_{\text {reg }}, W=\mathcal{E}-\left\{\bar{\varphi}\left(\mathcal{E}_{2}-W_{2}\right) \cup r\left(\varphi^{-1}\left(Z_{\text {sing }}\right)\right)\right\}$, alors $W$ est un ouvert dense de $\mathcal{E}$. Par l'hypothèse iii) du ThÉORÈme il existe $x \in \mathcal{C}_{L}$ tel que $r\left(y_{i}\right) \in W$, pour $1 \leq i \leq \sigma$, où $y_{1}, y_{2}, \ldots, y_{\sigma} \in \mathcal{C}_{L \widehat{K}}$ sont les points $\operatorname{de} \mathcal{C}_{L \widehat{K}}$ au-dessus de $x$; ce qui veut dire aussi que $r\left(y_{i}\right) \notin r\left(\rho^{-1}\left(Z_{\text {sing }}\right)\right)$ et $\bar{\varphi}^{-1}\left(r\left(y_{i}\right)\right)$ est régulier pour $1 \leq i \leq \sigma$.

r) Soit $U_{i}=r^{-1}\left(\mathcal{E}-\left\{r\left(y_{i}\right)\right\}\right)$. Alors il existe $h_{i} \in L \widehat{K}$ tel que $v_{y_{i}}\left(h_{i}\right)<0, v_{y_{j}}\left(h_{i}\right)=0$ pour $j \neq i$ et

$$
U_{i}=\left\{y \in \mathcal{C}_{L \widehat{K}} ; h_{i} \in \mathcal{O}_{\mathcal{C}_{L \widehat{K}}, y} \text { et }\left|h_{i}(y)\right| \leq 1\right\}
$$

( $v_{y_{i}}$ est la valuation discrète de $L \widehat{K} / \widehat{K}$ définie par le point $\left.y_{i}\right)$.

$\left.\gamma_{1}\right)$ Soit $\xi \in \bar{\varphi}^{-1}\left(\left\{r\left(y_{1}\right)\right\}\right)$, alors il existe $z \in \mathcal{C}_{L K_{2}}$ avec $\varphi(z)=y_{1}$ et $r_{2}(z)=\xi$ [Fr, exercice 4, p. 317]. Ainsi on peut choisir $z_{11}, z_{12}, \ldots, z_{1 \tau} \in$ $\varphi^{-1}\left(\left\{y_{1}\right\}\right)$ tels que $r_{2}\left(z_{1 i}\right) \neq r_{2}\left(z_{1 i^{\prime}}\right)$ pour $i \neq i^{\prime}$ et que

$$
\left\{r_{2}\left(z_{11}\right), \ldots, r_{2}\left(z_{1 \tau}\right)\right\}=\varphi^{-1}\left(\left\{r\left(y_{1}\right)\right\}\right) .
$$

Comme $\mathcal{E}$ est irréductible de dimension $1, \mathcal{E}-\left\{r\left(y_{1}\right)\right\}$ est affine, ainsi $\mathcal{E}_{2}-\left\{r_{2}\left(z_{11}\right), \ldots, r_{2}\left(z_{1 \tau}\right)\right\}=\bar{\varphi}^{-1}\left(\mathcal{E}-\left\{r\left(y_{1}\right)\right\}\right)$ est affine puisque $\bar{\varphi}$ est fini. La Proposition 3 ci-dessus montre alors qu'il existe $f_{1} \in L K_{2}$ tel que $f_{1} \in \mathcal{O}_{\mathcal{C}_{L K_{2}}}\left(\mathcal{C}_{L K_{2}}-\left\{z_{11}, \ldots, z_{1 \tau}\right\}\right)$, que $v_{z_{1 i}}\left(f_{1}\right)<0$ et que

$V_{1} \stackrel{\text { déf }}{=} r_{2}^{-1}\left(\mathcal{E}_{2}-\bar{\varphi}^{-1}\left(\left\{r\left(y_{1}\right)\right\}\right)\right)=\left\{z \in \mathcal{C}_{L K_{2}} ; f_{1} \in \mathcal{O}_{\mathcal{C}_{L K_{2}}, z}\right.$ et $\left.\left|f_{1}(z)\right| \leq 1\right\}$.

$\left.\gamma_{2}\right)$ Soient $G=\operatorname{Aut}\left(L K_{2} / L \widehat{K}\right), q=\left[L K_{2}: L \widehat{K}\right]_{\text {ins }}$, le degré d'inséparabilité, $g_{1}=\left(\prod_{\sigma \in G} f_{1}^{\sigma}\right)^{q}$; alors $g_{1} \in L \widehat{K}$. L'élément $\sigma \in G$ induit un automorphisme $\tilde{\sigma}$ de $\mathcal{C}_{L K_{2}} ;$ comme $V_{1}=\varphi^{-1}\left(U_{1}\right)$ où $U_{1}=r^{-1}\left(\mathcal{E}-\left\{r\left(y_{1}\right)\right\}\right)$, on a $\tilde{\sigma}\left(V_{1}\right)=V_{1}$, de plus on a $\left|f_{1}^{\sigma}(\tilde{\sigma}(z))\right|=\left|f_{1}(z)\right|$ si $f_{1} \in \mathcal{O}_{\mathcal{C}_{L K_{2}}, z}$. Il suit de cela que pour $z \in V_{1}$ on a $\left|f_{1}^{\sigma}(z)\right| \leq 1$ et que pour $z \notin V_{1}$ on a, ou 
bien $f_{1}^{\sigma} \notin \mathcal{O}_{\mathcal{C}_{L K_{2}}, z}$, ou bien $f_{1}^{\sigma} \in \mathcal{O}_{\mathcal{C}_{L K_{2}}, z}$ et $\left|f_{1}^{\sigma}(z)\right|>1$. Comme $\mathcal{C}_{L K_{2}}$ est régulier, on a facilement

$$
V_{1}=\left\{z \in \mathcal{C}_{L K_{2}} ; g_{1} \in \mathcal{O}_{\mathcal{C}_{L K_{2}}, z} \text { et }\left|g_{1}(z)\right| \leq 1\right\}
$$

Comme $V_{1}=\varphi^{-1}\left(U_{1}\right)$ et $g_{1} \in L \widehat{K}$ on déduit que

$$
U_{1}=\left\{y \in \mathcal{C}_{L \widehat{K}} ; g_{1} \in \mathcal{O}_{\mathcal{C}_{L \widehat{K}}, y} \text { et }\left|g_{1}(y)\right| \leq 1\right\},
$$

que $g_{1} \in \mathcal{O}_{\mathcal{C}_{L \widehat{K}}}\left(\mathcal{C}_{L \widehat{K}}-\left\{y_{1}\right\}\right)$ et que $v_{y_{1}}\left(g_{1}\right)<0$.

Il est facile de montrer qu'il existe $P(Z) \in K^{0}[Z]$ un polynôme unitaire tel que $\left|P\left(g_{1}\right)\left(y_{j}\right)\right|=1$ pour tout $y_{j} \in U_{1}$. Soit $h_{1}=P\left(g_{1}\right)$, on a donc $v_{y_{j}}\left(h_{1}\right)=0$ si $y_{j} \in U_{1}$; si $j \neq 1$ et $y_{j} \notin U_{1}$ on a $v_{y_{j}}\left(h_{1}\right) \geq 0$ et $\left|h_{1}\left(y_{j}\right)\right|>1$, ainsi $v_{y_{j}}\left(h_{1}\right)=0$; enfin $v_{y_{1}}\left(h_{1}\right)<0$. Il est facile de montrer que $U_{1}=\left\{y \in \mathcal{C}_{L \widehat{K}} ; h_{1} \in \mathcal{O}_{\mathcal{C}_{L \widehat{K}}, y}\right.$ et $\left.\left|h_{1}(y)\right| \leq 1\right\}$.

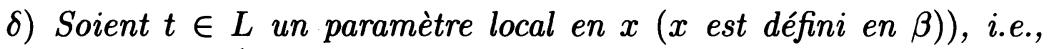
$v_{x}(t)=1, U=r^{-1}\left(\mathcal{E}-\left\{r\left(y_{1}\right), \ldots, r\left(y_{\sigma}\right)\right\}\right), Z=\left(\left(\mathcal{C}_{L}\right)_{(\widehat{K})}\right)_{\text {red. }}$. Alors il existe $h \in L \widehat{K}, \ell>0$ un entier, tels que $h \in \mathcal{O}_{Z}\left(Z-\rho\left(\left\{y_{1}, y_{2}, \ldots, y_{\sigma}\right\}\right)\right)$, alors $v_{y_{i}}(h)=v_{y_{i}}\left(t^{-\ell}\right)$ pour $1 \leq i \leq \sigma$ et

$$
U=\left\{y \in \mathcal{C}_{L \widehat{K}} ; h \in \mathcal{O}_{\mathcal{C}_{L \widehat{K}}, y} \text { et }|h(y)| \leq 1\right\} \text {. }
$$

Par $\gamma)$ il existe $h_{1}, \ldots h_{\sigma} \in L \widehat{K}$ tels que $h_{i} \in \mathcal{O}_{\mathcal{C}_{L \widehat{K}}}\left(\mathcal{C}_{L \widehat{K}}-\left\{y_{i}\right\}\right)$, que $v_{y_{i}}\left(h_{i}\right)<0$ et que $U_{i}=\left\{y \in \mathcal{C}_{L \widehat{K}} ; h_{i} \in \mathcal{O}_{\mathcal{C}_{L \widehat{K}}, y}\right.$ et $\left.\left|h_{i}(y)\right| \leq 1\right\}$. Il existe des entiers positifs, $n_{1}, n_{2}, \ldots, n_{\sigma}$ tels que

$$
\frac{v_{y_{1}}\left(h_{1}^{n_{1}}\right)}{v_{y_{1}}(t)}=\cdots=\frac{v_{y_{\sigma}}\left(h_{\sigma}^{n_{\sigma}}\right)}{v_{y_{\sigma}}(t)}
$$

et que $v_{y_{1}}\left(h_{1}^{n_{1}}\right) / v_{y_{1}}(t)$ soit un entier $-d$ négatif. Soit $h^{\prime}=\prod_{i} h_{i}^{n_{i}}$, on a $v_{y_{i}}\left(h^{\prime}\right)=-d v_{y_{i}}(t)$, pour $1 \leq i \leq \sigma$ et par $\left.\gamma\right)$,

$$
U=\bigcap_{i} U_{i}=\left\{y \in \mathcal{C}_{L \widehat{K}} ; h^{\prime} \in \mathcal{O}_{\mathcal{C}_{L \widehat{K}}, y} \text { et }\left|h^{\prime}(y)\right| \leq 1\right\}
$$

$h^{\prime} \in \mathcal{O}_{\mathcal{C}_{L \widehat{K}}}\left(\mathcal{C}_{L \widehat{K}}-\left\{y_{1}, \ldots, y_{\sigma}\right\}\right)$.

Soit $\left\{u_{1}, u_{2}, \ldots, u_{\nu}\right\}=\rho^{-1}\left(Z_{\text {sing }}\right)$, par $\left.\beta\right)$ on a $\rho^{-1}\left(Z_{\text {sing }}\right) \subset U$; ainsi $\left|h^{\prime}\left(u_{i}\right)\right| \leq 1$. Soit $P_{i}(Y)=\operatorname{irr}\left(h^{\prime}\left(u_{i}\right), \widehat{K}, Y\right)$, alors $P_{i}\left(h^{\prime}\right)\left(u_{i}\right)=0$. Il suit du Lemme 1, ci-après, qu'il existe $N>0$ avec

$$
h \stackrel{\text { déf }}{=}\left(\prod_{i} P_{i}\left(h^{\prime}\right)\right)^{N} \in \mathcal{O}_{Z, \rho\left(u_{i}\right)} \quad \text { pour } 1 \leq i \leq \nu .
$$

TOME $116-1988-\mathrm{N}^{\circ} 1$ 
Comme $\prod_{i} P_{i}(Y)^{N} \in \widehat{K}^{0}[Y]$ est unitaire on a facilement

$$
U=\left\{y \in \mathcal{C}_{L \widehat{K}} ; h \in \mathcal{O}_{\mathcal{C}_{L \widehat{K}}, y} \text { et }|h(y)| \leq 1\right\},
$$

$h \in \mathcal{O}_{Z}\left(Z-\rho\left(\left\{y_{1}, \ldots, y_{\sigma}\right\}\right)\right)$ et $v_{y_{i}}(h)=-\ell v_{y_{i}}(t)$ où $\ell>0$ est entier.

$\epsilon)$ Soit $\mathcal{L}$ (resp. $\mathcal{L}_{1}$ ) le faisceau inversible de $\mathcal{C}_{L}$ (resp. $Z$ ) défini par $\mathcal{L}_{x^{\prime}}=\mathcal{O}_{\mathcal{C}_{L}, x^{\prime}}$, si $x^{\prime} \neq x, \mathcal{L}_{x}=t^{-1} \mathcal{O}_{\mathcal{C}_{L}, x}$ (resp. $\mathcal{L}_{1 y}=\mathcal{O}_{Z, y}$ si $y \neq y_{i}$ et $\left.\mathcal{L}_{1 y_{i}}=t^{-1} \mathcal{O}_{Z, y_{i}}\right)$. Alors pour tout $n \gg 0$ l'homomorphisme canonique $\widehat{K} \otimes_{K} \mathcal{L}^{n}\left(\mathcal{C}_{L}\right) \rightarrow \mathcal{L}_{1}^{n}(Z)$ est surjectif.

Il existe $n>0$ et $T_{1} \in \mathcal{L}^{n}\left(\mathcal{C}_{L}\right)$ avec

$$
U=\left\{y \in \mathcal{C}_{L \widehat{K}} ; T_{1} \in \mathcal{O}_{\mathcal{C}_{L \widehat{K}}, y} \text { et }\left|T_{1}(y)\right| \leq 1\right\} .
$$

$\left.\epsilon_{1}\right)$ Le faisceau inversible $\mathcal{L} \operatorname{sur} \mathcal{C}_{L}$ est ample, donc aussi

$$
\mathcal{L}_{(\widehat{K})} \stackrel{\text { déf }}{=} \mathcal{L} \otimes \mathcal{O}_{\mathcal{C}_{L}}\left(\mathcal{O}_{\mathcal{C}_{L}} \otimes \widehat{K}\right)
$$

défini sur $\left(\mathcal{C}_{L}\right)_{(\widehat{K})}$. Soit $\mathcal{N}$ le faisceau des nilpotents de $\mathcal{O}_{\left(\mathcal{C}_{L}\right)}$. . De la suite exacte $0 \rightarrow \mathcal{N} \rightarrow \mathcal{O}_{\left(\mathcal{C}_{L}\right)}(\widehat{K})$ $\rightarrow \mathcal{O}_{Z} \rightarrow 0$, on déduit par tensorisation la suite exacte $0 \rightarrow \mathcal{N} \otimes \mathcal{L}_{(\widehat{K})}^{n} \rightarrow \mathcal{L}_{(\widehat{K})}^{n} \rightarrow \mathcal{L}_{1}^{n} \rightarrow 0$ ce qui donne l'exactitude de la suite de cohomologie,

$$
H^{0}\left(\left(\mathcal{C}_{L}\right)_{(\widehat{K})}, \mathcal{L}_{(\widehat{K})}^{n}\right) \rightarrow H^{0}\left(Z, \mathcal{L}_{1}^{n}\right) \rightarrow H^{1}\left(\left(\mathcal{C}_{L}\right)_{(\widehat{K})}, \mathcal{N} \otimes \mathcal{L}_{(\widehat{K})}^{n}\right) .
$$

Comme $\mathcal{L}_{(\widehat{K})}$ est ample, on a $H^{1}\left(\left(\mathcal{C}_{L}\right)_{(\widehat{K})}, \mathcal{N} \otimes \mathcal{L}_{(\widehat{K})}^{n}\right)=0$ pour $n \gg 0$. Il suit de cela que l'homomorphisme canonique $\widehat{K} \otimes_{K} \mathcal{L}\left(\mathcal{C}_{L}\right) \rightarrow \mathcal{L}_{1}^{n}(Z)$ est surjectif.

$\epsilon_{2}$ ) Quitte à changer $h$ défini par $\delta$ ), en une puissance de $h$, en utilisant $\left.\epsilon_{1}\right)$ on a $h \in \mathcal{L}_{1}^{n}(Z)=\widehat{K} \mathcal{L}^{n}\left(\mathcal{C}_{L}\right)$ avec

$$
\begin{gathered}
v_{y_{i}}(h)=v_{y_{i}}\left(t^{-n}\right) \quad \text { pour } 1 \leq i \leq \sigma, \\
U=\left\{y \in \mathcal{C}_{L \widehat{K}} ; h \in \mathcal{O}_{\mathcal{C}_{L \widehat{K}}, y} \text { et }|h(y)| \leq 1\right\} .
\end{gathered}
$$

Ainsi il existe avec $h=\sum \lambda_{i} w_{i}$

$$
w_{1}, w_{2}, \ldots, w_{\alpha} \in \mathcal{L}^{n}\left(\mathcal{C}_{L}\right), \quad \lambda_{1}, \ldots, \lambda_{\alpha} \in \widehat{K} .
$$

Il existe $\theta_{1}, \theta_{2}, \ldots, \theta_{\beta} \in \mathcal{O}_{\mathcal{C}_{L}, x}, K$-linéairement indépendants tels que l'application canonique $\bigoplus_{\ell=1}^{\beta} K \theta_{\ell} \rightarrow K(x)=\mathcal{O}_{\mathcal{C}_{L}, x} / \mathfrak{m}_{x}$ soit bijective. 
Il suit que $w_{i}=\sum_{m=1}^{m=n} A_{m}^{i} / t^{m}+w_{i}^{\prime}$ avec $A_{m}^{i} \in \bigoplus_{\ell} K \theta_{\ell}, w_{i}^{\prime} \in \mathcal{O}_{\mathcal{C}_{L}, x}$, et par (1), (2) on a

$$
\sum_{i} \lambda_{i} A_{n}^{i}\left(y_{j}\right) \neq 0 \quad \text { pour } 1 \leq j \leq \sigma .
$$

Il existe un affinoïde (admissible) $W_{1}^{j}$ de $\mathcal{C}_{L \widehat{K}}^{\text {an }}$ tel que $A_{m}^{i}, w_{i}^{\prime}$ soient réguliers sur $W_{1}^{j}$ pour $1 \leq i \leq \alpha, 1 \leq m \leq n$ et que $y_{j} \in W_{1}^{j}, y_{j} \notin W_{1}^{j^{\prime}}$ pour $j^{\prime} \neq j$. Comme $\sum_{i} \lambda_{i} A_{n}^{i}\left(y_{j}\right) \neq 0$ pour $1 \leq j \leq \sigma$, il existe un admissible affinoïde $W_{2}^{j} \subset W_{1}^{j}$ tel que $y_{j} \in W_{2}^{j}$ pour $1 \leq j \leq \sigma$ et que pour tout $y \in W_{2}^{j}$

$$
\left|\sum_{i} \lambda_{i} A_{n}^{i}(y)\right|=\left|\sum_{i} \lambda_{i} A_{n}^{i}\left(y_{j}\right)\right| \neq 0
$$

Soient $\pi \in \widehat{K}, W_{3}^{j}=\left\{y \in W_{2}^{j} ;|t(y)| \leq|\pi|\right\}$, en choisissant $|\pi|$ assez petit on a

$$
\left|\frac{\sum_{i} \lambda_{i} A_{n}^{i}(y)}{t^{n}(y)}\right|>\left|\frac{\sum_{i} \lambda_{i} A_{m}^{i}(y)}{t^{m}(y)}\right|, \quad\left|\frac{\sum_{i} \lambda_{i} A_{n}^{i}(y)}{t^{n}(y)}\right|>\left|\sum_{i} \lambda_{i} w_{i}^{\prime}(y)\right|,
$$

pour tout $y \in W_{3}^{j}-\left\{y_{j}\right\}$ et $1 \leq m<n$.

Il existe un affinoïde $W^{\prime} \operatorname{de} \mathcal{C}_{L \widehat{K}}^{\text {an }}$ tel que $y_{j} \notin W^{\prime}$ pour $1 \leq j \leq \sigma$ et $W^{\prime} \cup\left(\bigcup_{1 \leq j \leq \sigma} W_{3}^{j}\right)=\mathcal{C}_{L \widehat{K}}^{\text {an }}$.

Comme $w_{i}$ est régulier sur $W^{\prime}$, pour $\mu_{i} \in K$ et $\left|\mu_{i}-\lambda_{i}\right|$ assez petit on a pour tout $y \in W^{\prime}$

$$
\left|\sum_{i} \lambda_{i} w_{i}(y)-\sum_{i} \mu_{i} w_{i}(y)\right|<1
$$

Comme $w_{i}^{\prime}$ est régulier sur $W_{3}^{j}$, pour $\mu_{i} \in K$ et $\left|\mu_{i}-\lambda_{i}\right|$ assez petit on a pour tout $y \in W_{3}^{j}$

$$
\left|\sum_{i} \lambda_{i} w_{i}^{\prime}(y)-\sum_{i} \mu_{i} w_{i}^{\prime}(y)\right|<1
$$

Comme $A_{m}^{i}$ est régulier sur $W_{3}^{j}$ il suit de (5), (3) et (7) que pour $\mu_{i} \in K$ et $\left|\lambda_{i}-\mu_{i}\right|$ assez petit on a

$$
\left|\sum \lambda_{i} A_{n}^{i}(y)\right|=\left|\sum \lambda_{i} A_{n}^{i}\left(y_{j}\right)\right|=\left|\sum \mu_{i} A_{n}^{i}(y)\right|
$$

TOME $116-1988-\mathrm{N}^{\circ} 1$ 
pour tout $y \in W_{3}^{j}$ et que pour tout $y \in W_{3}^{j}-\left\{y_{j}\right\}$ et $1 \leq m<n$ on a

$$
\left|\frac{\sum_{i} \mu_{i} A_{n}^{i}(y)}{t^{n}(y)}\right|>\left|\frac{\sum_{i} \mu_{i} A_{m}^{i}(y)}{t^{m}(y)}\right|, \quad\left|\frac{\sum_{i} \mu_{i} A_{n}^{i}(y)}{t^{n}(y)}\right|>\left|\sum_{i} \mu_{i} w_{i}^{\prime}(y)\right| .
$$

Il suit de (8) que $v_{y_{j}}\left(\sum_{i} \mu_{i} w_{i}\right)=v_{y_{j}}\left(t^{-n}\right)$ et que pour $y \in W_{3}^{j}-\left\{y_{j}\right\}$

$$
\left|\sum \lambda_{i} w_{i}(y)\right|=\left|\sum \mu_{i} w_{i}(y)\right|
$$

Soit $T_{1}=\sum_{i} \mu_{i} w_{i} \in \mathcal{L}^{n}\left(\mathcal{C}_{L}\right) \subset L$, il suit donc de (6), (9) que $U=\left\{y \in \mathcal{C}_{L \widehat{K}} ; T_{1} \in \mathcal{O}_{\mathcal{C}_{L \widehat{K}}, y}\right.$ et $\left.\left|T_{1}(y)\right| \leq 1\right\}$. Ce qui montre $\left.\delta_{2}\right)$.

$\varphi)$ L'élément $T_{1} \in L$ défini en $\gamma$ ) est transcendant sur $\widehat{K}$ et $|\cdot|$ est l'unique prolongement à $L$ de la valeur absolue de Gauss sur $K\left(T_{1}\right)$ associée à $T_{1}$ et $\grave{a}|\cdot|_{0}$.

Comme $v_{y_{j}}\left(T_{1}\right)<0$ par la formule $(9)$ de $\gamma_{2}$ ) on a bien $T_{1}$ qui est transcendant sur $\widehat{K}$.

Comme $U \stackrel{\text { déf }}{=} r^{-1}\left(\mathcal{E}-\left\{r\left(y_{1}\right), \ldots, r\left(y_{\sigma}\right)\right\}\right)$ où $\mathcal{E}-\left\{r\left(y_{1}\right), \ldots, r\left(y_{\sigma}\right)\right\}$ est affine et irréductible, l'ouvert $U$ est affinoïde et on a

$$
\bar{U}^{c}=r(U)=\mathcal{E}-\left\{r\left(y_{1}\right), \ldots, r\left(y_{\sigma}\right)\right\}
$$

D'autre part $\left.U=U\left(T_{1}\right)(\operatorname{par} \gamma)\right)$ et la Proposition $1, \S 1.2$, montre que la valeur absolue de Gauss sur $\widehat{K}\left(T_{1}\right)$ associée à $T_{1}$ et à $|\cdot|_{0}$ admet un prolongement unique à $L \widehat{K}$ parce que $\bar{U}^{c}={\overline{U\left(T_{1}\right)}}^{c}$ est irréductible.

Ainsi $\varphi$ ) suit de la Proposition 2, § 1.3.

Corollaire 1. - Soient $L / K$ un corps de fonctions d'une variable dont le corps des constantes est purement inséparable sur $K,\left\{|\cdot|_{j}\right\}_{1 \leq j \leq s}$ des valeurs absolues sur $L$ qui coïncident sur $K$ en une valeur absolue notée $|\cdot|$. On suppose que $\left(\overline{L,|\cdot|_{j}}\right) /(\overline{K,|\cdot|})$ est un corps de fonctions d'une variable pour $1 \leq j \leq s$ et que $\left(L / K,|\cdot|_{j}\right)$ satisfait l'une des propriétés équivalentes i), ii), iii), du ThÉORÈme 1 pour $1 \leq j \leq s$. Alors il existe $T \in L$ transcendant sur $K$ tel que $\left\{|\cdot|_{j}\right\}_{1 \leq j \leq s}$ soient exactement les prolongements à $L$ de la valeur absolue de Gauss sur $K(T)$ associée à $|\cdot|$ et $\grave{a} T$.

Démonstration.

a) Il existe $Z_{i} \in L \subset L \widehat{K}$ tel que $\left|Z_{i}-1\right|_{j}<1$ pour $j \neq i$, que $Z_{i}$ soit transcendant sur $\widehat{K}$, que la restriction de $|\cdot|_{i} \grave{a} \widehat{K}\left(Z_{i}\right)$ soit la valeur absolue de Gauss $|\cdot|_{g}$ sur $\widehat{K}\left(Z_{i}\right)$ associée à $Z_{i}$ et $|\cdot|$ et que 
$|\cdot|_{i}$ soit l'unique prolongement de $|\cdot|_{g} \grave{a} L \widehat{K}$ (on note aussi $|\cdot|_{j}$ l'unique prolongement de $|\cdot|_{j}$ à $L \widehat{K}$, Proposition $\left.2, \S 1.3\right)$.

Il existe $T_{i} \in L$ transcendant sur $\widehat{K}$ tel que la restriction de $|\cdot|_{i}$ à $\widehat{K}\left(T_{i}\right)$ soit la valeur absolue de Gauss $|\cdot|_{g}$ associée à $T_{i}$ et $|\cdot|$, et que $|\cdot|_{i}$ soit l'unique prolongement de $\mid \cdot l_{g}$ à $L \widehat{K}$ (c'est l'hypothèse du Corollaire et la Proposition 2, $\S 1.3)$. Soit $j \neq i$ avec $\left|T_{i}\right|_{j} \leq 1$, la restriction de $|\cdot|_{j}$ à $\widehat{K}\left(T_{i}\right)$ n'est pas $|\cdot|_{g}$; ainsi il existe $P_{j}(Y) \in K^{0}[Y]$ unitaire tel que $\left|P_{j}\left(T_{i}\right)\right|<1$. Soit $Q=\prod P_{j}$, le produit est pris sur les $j \neq i$ tels que $\left|T_{i}\right|_{j} \leq 1$, alors on a $\left|Q\left(T_{i}\right)\right|_{j} \neq 1$ pour tout $j \neq 1$; si $\left|T_{i}\right|_{j}>1$ on a bien $\left|Q\left(T_{i}\right)\right|>1$. Soit $R(Y)=\left(1+3 Q+Q^{2}\right) /\left(1+2 Q+Q^{2}\right)$, alors on a

$$
\left|R\left(T_{i}\right)-1\right|_{j}<1 \quad \text { pour } j \neq 1 \text {. }
$$

Soit $Z_{i}=R\left(T_{i}\right)$, on a $\left[\widehat{K}\left(T_{i}\right): \widehat{K}\left(Z_{i}\right)\right]=\operatorname{deg}\left(Q^{2}\right)$, l'image de $Z_{i}$ dans $\left(\overline{K\left(T_{i}\right),|\cdot|_{i}}\right)$ est transcendante sur $\bar{K}$ et on a

$$
\left[\left(\overline{\left(T_{i}\right),|\cdot|_{i}}\right):\left(\overline{K\left(Z_{i}\right),|\cdot|_{i}}\right)\right]=\operatorname{deg}\left(Q^{2}\right)
$$

Il suit de cela que $|\cdot|_{g}$ restreint à $K\left(Z_{i}\right)$ est la valeur absolue de Gauss $|\cdot|_{g}^{\prime}$ associée à $Z_{i}$ et $|\cdot|$, que $|\cdot|_{g}$ est l'unique prolongement de $|\cdot|_{g}^{\prime}$ à $\widehat{K}\left(T_{i}\right)$ et que $|\cdot|_{i}$ est l'unique prolongement de $|\cdot|_{g}^{\prime}$ à $L$.

$\beta$ ) Soit $Z=Z_{1} Z_{2} \ldots Z_{s}$. Alors $Z$ est transcendant sur $K$ et $\left\{|\cdot|_{i}\right\}_{1 \leq i \leq s}$ est exactement l'ensemble des prolongements à $L$ de la valeur absolue de Gauss sur $K(Z)$ associée à $Z$ et $\grave{a}|\cdot|$.

Soient $n_{i}=\left[L \widehat{K}: \widehat{K}\left(Z_{i}\right)\right], D_{i}$ le diviseur des pôles de $Z_{i}$ dans $L \widehat{K} / \widehat{K}$, alors $n_{i}=\operatorname{deg} D_{i}$. Soit $D$ le diviseur des pôles de $Z$ dans $L \widehat{K} / \widehat{K}$, alors on a $[L \widehat{K}: \widehat{K}(Z)]=\operatorname{deg} D$ et comme $\operatorname{deg} D \leq \sum_{i} \operatorname{deg} D_{i}$, on a

$$
[L \widehat{K}: \widehat{K}(Z)] \leq n_{1}+n_{2}+\cdots+n_{s} .
$$

Par $\alpha$ ) on a [Ma1, $\S 1.2$, corollaire 2 de la proposition 5]

$$
\left[\left(L \widehat{K},|\cdot|_{i}\right)^{\wedge}:\left(\widehat{K}\left(Z_{i}\right),|\cdot|_{i}\right)^{\wedge}\right]=\left[L \widehat{K}: \widehat{K}\left(Z_{i}\right)\right]=n_{i}
$$

Comme $\left(\overline{\widehat{K}\left(Z_{i}\right),|\cdot|_{i}}\right)=\bar{K}\left(\bar{Z}_{i}\right)=\left(\overline{\widehat{K}(Z),|\cdot|_{i}}\right)$, on a

$$
\left[\left(\overline{\left(\widehat{L},|\cdot|_{i}\right.}\right):\left(\overline{\widehat{K}(Z),|\cdot|_{i}}\right)\right]=\left[\left(\overline{L \widehat{K},|\cdot|_{i}}\right):\left(\overline{\widehat{K}\left(Z_{i}\right),|\cdot|_{i}}\right)\right]
$$

En plus

$$
e\left(L \widehat{K} / \widehat{K}(Z),|\cdot|_{i}\right)=e\left(L \widehat{K} / \widehat{K}\left(Z_{i}\right),|\cdot|_{i}\right)=e\left(L \widehat{K} / \widehat{K},|\cdot|_{i}\right)
$$

TOME $116-1988-\mathrm{N}^{\circ} 1$ 
Enfin

$$
\begin{aligned}
d\left(\left(L \widehat{K},|\cdot|_{i}\right)^{\wedge} /\left(\widehat{K}(Z),|\cdot|_{i}^{\wedge}\right)\right. & =d\left(\left(L \widehat{K},|\cdot|{ }_{i}\right)^{\wedge} /\left(\widehat{K}\left(Z_{i}\right),|\cdot|_{i}\right)^{\wedge}\right) \\
& =d\left(L \widehat{K} / \widehat{K},|\cdot|_{i}\right)
\end{aligned}
$$

[Ma1, § 1.4.2, corollaire 1 de la proposition 8]; $e(L / K,|\cdot|)$ est l'indice de ramification de $L / K$ i.e.,

$$
e(L / K,|\cdot|)=\frac{\left|L^{\times}\right|}{\left|K^{\times}\right|}
$$

$d(L / K,|\cdot|)$ est le défaut de $L / K$, i.e.

$$
d(L / K,|\cdot|)=\frac{\left[\widehat{L}: K(T)^{\wedge}\right]}{e(L / K,|\cdot|) \cdot[\bar{L}: \overline{K(T)}]}
$$

pour $T \in L$ avec $|T|=1$ et résiduellement transcendant [Ma1, corollaire $1, \S 1.4 .2]$.

Il suit de (2), (3), (4) que

$$
\left[\left(L \widehat{K},|\cdot|_{i}\right)^{\wedge}:\left(\widehat{K}(Z),|\cdot|_{i}\right)^{\wedge}\right]=\left[\left(L \widehat{K},|\cdot|_{i}\right)^{\wedge}:\left(\widehat{K}\left(Z_{i}\right),|\cdot|_{i}\right)^{\wedge}\right] .
$$

Soient $|\cdot|_{1},|\cdot|_{2}, \ldots,|\cdot|_{s},|\cdot|_{s+1}, \ldots,|\cdot|_{s+s^{\prime}}$ les valeurs absolues de $L \widehat{K}$ qui prolongent la valeur absolue de Gauss sur $\widehat{K}(Z)$ associée à $Z$ et $|\cdot|$. On a

$$
[L \widehat{K}: \widehat{K}(Z)] \geq \sum_{i=1}^{s+s^{\prime}}\left[\left(L \widehat{K},|\cdot|_{i}\right)^{\wedge}:\left(\widehat{K}(Z),|\cdot|_{i}\right)^{\wedge}\right]
$$

(en fait on a une égalité, [Ma1, $\S 1.2$, corollaire 2 de la proposition 5]). Il suit de $(1),(2),(6)$ et $(7)$ que $s^{\prime}=0$. Ainsi $\beta$ ) est montré en utilisant la Proposition 2, $\S 1.3$.

Corollaire 2 [La 2]. - Soient $(L / K,|\cdot|)$ un corps de fonctions d'une variable valué tel que $\bar{L} / \bar{K}$ soit un corps de fonctions d'une variable. On suppose les propriétés suivantes satisfaites.

1) Le corps des constantes de $L / K$ est $K$, celui de $\bar{L} / \bar{K}$ est $\bar{K}$.

2) genre $(L / K)=$ genre $(\bar{L} / \bar{K})$.

3) $e(L / K,|\cdot|)=d(L / K,|\cdot|)=1$. Alors $(L / K,|\cdot|)$ satisfait les propriétés i), ii), iii), du THÉORÈmE 1.

Démonstration. - Par la proprosition 6, $\S 7.1$ de [Ma2], le corps de fonctions valué $(L \widehat{K} / \widehat{K},|\cdot|)$ satisfait 1$), 2), 3)$. On conserve les notations 
de la démonstration du ThÉorème 1, iii) implique i), ainsi il existe $T \in$ $L \widehat{K}$ qui définit une réduction analytique $r: \mathcal{C}_{L \widehat{K}} \rightarrow \mathcal{E}$ où $\mathcal{E}$ est irréductible. Il suit de 3) que $\chi\left(\mathcal{O}_{\mathcal{C}_{L \widehat{K}}}\right)=\chi\left(\mathcal{O}_{\mathcal{E}}\right)$ où $\mathcal{O}_{\mathcal{C}_{L \widehat{K}}}\left(\right.$ resp. $\left.\mathcal{O}_{\mathcal{E}}\right)$ est le faisceau structural de $\mathcal{C}_{L \widehat{K}}$ (resp. $\left.\mathcal{E}\right), \chi$ est la caractéristique d'Euler-Poincaré, on a donc 1-genre $(L / K)=\operatorname{dim}_{\bar{K}} H^{0}\left(\mathcal{E}, \mathcal{O}_{\mathcal{E}}\right)-\operatorname{dim}_{\bar{K}} H^{1}\left(\mathcal{E}, \mathcal{O}_{\mathcal{E}}\right)$. Soit $\mathcal{E}^{\prime}$ la normalisation de $\mathcal{E}$, on a $\mathcal{E}^{\prime}=\mathcal{C}_{\bar{L}}$, par 1) $H^{0}\left(\mathcal{E}, \mathcal{O}_{\mathcal{E}}\right)=H^{0}\left(\mathcal{E}^{\prime}, \mathcal{O}_{\mathcal{E}^{\prime}}\right)=\bar{K}$, par 2) on déduit que $\operatorname{dim}_{\bar{K}} H^{1}\left(\mathcal{E}, \mathcal{O}_{\mathcal{E}}\right)=\operatorname{dim}_{\bar{K}} H^{1}\left(\mathcal{E}^{\prime}, \mathcal{O}_{\mathcal{E}^{\prime}}\right)$, ce qui montre que $\mathcal{E}$ est régulier.

Soit $x \in \mathcal{C}_{L}$ (quelconque), $y_{1}, y_{2}, \ldots, y_{\sigma} \in \mathcal{C}_{L \widehat{K}}$ les points de $\mathcal{C}_{L \widehat{K}}$ audessus de $x$; par la Proposition 3 , il existe $h_{i} \in L \widehat{K}$ tel que $v_{y_{i}}\left(h_{i}\right)<0$, $v_{y_{j}}\left(h_{i}\right)=0$ pour $j \neq i$ et

$$
r^{-1}\left(\mathcal{E}-r\left(y_{i}\right)\right)=\left\{z \in \mathcal{C}_{L \widehat{K}} ; h_{i} \in \mathcal{O}_{\mathcal{C}_{L \widehat{K}}, z} \text { et }\left|h_{i}(z)\right| \leq 1\right\} .
$$

Ainsi $\delta), \epsilon), \varphi$ ), de la démonstration de la partie iii) implique i) du THÉORÈme 1 montrent qu'il existe $T \in L$ satisfaisant la propriété i) du THÉORÈME 1.

Remarque. - La démonstration de E. LAMPRECht dans le cas où le corps $K$ est muni d'une valeur absolue discrète s'adapte pour démontrer le Corollaire 2. En revanche cette technique ne pourrait, à notre avis, conduire au THÉORÈME 1.

\section{Prolongement de la valeur absolue de Gauss et propriété de Skolem}

Définition 3. - Soient $(K,|\cdot|)$ un corps valué, $\widehat{K}$ le complété de $K, \widehat{K}^{\text {alg }}$ la clôture algébrique de $\widehat{K}, X$ une variété algébrique sur $K$, géométriquement irréductible, $\mathcal{F}=\left\{f_{1}, \ldots, f_{r}\right\}$ une partie finie de $\mathcal{R}(X)$, l'anneau des fonctions rationnelles sur $X$; i.e., $\mathcal{R}(X)=\lim _{\longrightarrow} \mathcal{O}_{X}(U)$ où la limite inductive est prise sur tous les ouverts non vides. Si $x \in X$ est un point (fermé), on désigne par $f_{i}(x)$ l'image de $f_{i}$ dans $K(x) \stackrel{\text { déf }}{=} \mathcal{O}_{X, x} / \mathfrak{m}_{x}$. Soit

$$
U(\mathcal{F}) \stackrel{\text { déf }}{=}\left\{y \in X_{(\widehat{K})} ; f_{i} \in \mathcal{O}_{X_{(\widehat{K})}, y} \quad \text { et } \quad\left|f_{i}(y)\right| \leq 1 \text { pour } 1 \leq i \leq r\right\},
$$

où $|\cdot|$ est l'unique valeur absolue de

$$
\widehat{K}(y) \stackrel{\text { déf }}{=} \frac{\mathcal{O}_{X}, y}{\mathfrak{M}_{y}}
$$

TOME $116-1988-\mathrm{N}^{\circ} 1$ 
prolongeant celle de $\widehat{K}, f_{i}(y)$ est l'image canonique de $f_{i}$ dans $\widehat{K}(y)$, et $X_{(\widehat{K})}$ désigne la variété algébrique sur $\widehat{K}$ obtenue par extension des scalaires à $\widehat{K}$.

Alors on dit que le triplet $\left\{(K,|\cdot|) ; X_{(\widehat{K})} ; \mathcal{F}\right\}$ satisfait la propriété de Skolem (locale) si $U(\mathcal{F})=\emptyset$ ou si $U(\mathcal{F}) \neq \emptyset$ implique qu'il existe $x \in X$ (un point fermé) tel que $f_{i}(x)$ soit entier sur $K^{0}$ pour $1 \leq i \leq r\left(K^{0}\right.$ est l'anneau de valuation de $K$ ); cette dernière condition veut aussi dire que $\mid \sigma\left(f_{i}(x \downarrow) \mid \leq 1\right.$ pour tout $K$-homomorphisme $\sigma: K(x) \rightarrow \widehat{K}^{\text {alg }}$ (où $|\cdot|$ est l'unique valeur absolue de $\widehat{K}^{\text {alg }}$ prolongeant celle de $\left.\widehat{K}\right)$; elle veut aussi dire que tout point de $X_{(\widehat{K})}$ au-dessus de $x$ est dans $U(\mathcal{F})$.

On dit que le couple $\{(K,|\cdot|) ; X\}$ satisfait la propriété de Skolem (locale) si pour toute partie finie $\mathcal{F}$ de $\mathcal{R}(X)$ le triplet $\{(K,|\cdot|) ; X ; \mathcal{F}\}$ satisfait la propriété de Skolem.

On dit que $(K,|\cdot|)$ satisfait la propriété de Skolem (locale) si pour toute variété $X$ géométriquement irréductible, le couple $\{(K,|\cdot|) ; X\}$ satisfait la propriété de Skolem.

Remarque. - La notion de propriété de Skolem est en général utilisée et définie comme une notion globale, i.e., c'est une propriété satisfaite par l'ensemble des valeurs absolues d'un corps $K$ sauf pour une partie finie de l'ensemble [Ro2], [Ru], [Ca, Ro].

Les propositions qui suivent sont élémentaires, en revanche le théorème est loin d'être facile.

Proposition 4. - Soient $(K,|\cdot|)$ un corps valué, $X$ une variété algébrique, géométriquement irréductible, $S=\{y \in X ;|\cdot|$ admet un unique prolongement à $\left.K(y)=\mathcal{O}_{X, y} / \mathfrak{M}_{y}\right\}$; on suppose que $S$ est dense dans $X_{(\widehat{K})}$ pour la topologie induite sur $X_{(\widehat{K})}$ par celle de $\widehat{K} L$ (clairement $S$ s'injecte dans $\left.X_{(\widehat{K})}\right)$. Alors le couple $\{(K,|\cdot|) ; X\}$ satisfait la propriété de Skolem locale. (Voir [Pz, §3, Corollaire 1]).

Proposition $4^{\prime}$. - Soient $(K,|\cdot|)$ un corps valué, $\mathcal{C}$ une courbe projective, non singulière, géométriquement irréductible sur $K$, de genre zéro. Alors $\{(K,|\cdot|) ; \mathcal{C}\}$ satisfait la propriété de Skolem (locale). (Voir $[\mathrm{Pz}, \S 3$, Proposition 7]).

Corollaire 1. - Soient $(K,|\cdot|)$ un corps valué, $X$ une variété algébrique sur $K$, birationnelle à $\mathrm{A}_{K}^{n}$. Alors le couple $\{(K,|\cdot|) ; X\}$ satisfait la propriété de Skolem (locale). (Voir [Pz, §3, Proposition 6]).

Corollaire 2. - Un corps $(K,|\cdot|)$ hensélien satisfait la propriété de Skolem (locale). (Voir $[\mathrm{Pz}, \S 3$, Corollaire 2]). 
ThÉorème ([Ru], [Ro2], [Mo-Ba, Szp], [Mo-Ba]). - Soit $K$ un corps valué avec une valeur absolue ultramétrique discrète (i.e., $\left.\left|K^{\times}\right| \simeq \mathbf{Z}\right)$ dont le corps résiduel $\bar{K}$ est algébrique sur un corps fini. Alors $(K,|\cdot|)$ satisfait la propriété de Skolem (locale).

Proposition 5. - Soit $(K,|\cdot|)$ un corps valué. Alors les propriétés suivantes sont équivalentes.

i) Le corps valué $(K,|\cdot|)$ satisfait la propriété de Skolem,

ii) pour tout corps de fonctions d'une variable $L / K$ dont le corps des constantes est purement inséparable sur $K$, le couple $\left\{(K,|\cdot|) ; \mathcal{C}_{L}\right\}$ satisfait la propriété de Skolem.

Démonstration. - On a clairement i) implique ii). Montrons ii) implique i). Soient $X$ une variété algébrique sur $K$, géométriquement irréductible, $\mathcal{F}=\left\{f_{1}, \ldots, f_{r}\right\}$ une partie finie de $\mathcal{R}(X)$ telle que $U(\mathcal{F})$ soit non vide; il s'agit de montrer que $\{(K,|\cdot|) ; X ; \mathcal{F}\}$ satisfait la propriété de Skolem. Soit $Y$ un ouvert non vide de $X$ tel que $f_{1}, \ldots, f_{r}$ soient réguliers sur $Y$, il est équivalent de montrer que $\{(K,|\cdot|) ; Y ; \mathcal{F}\}$ satisfait la propriété de Skolem (Corollaire 1 du Lemme 2). On a $U(\mathcal{F}) \cap Y_{(\widehat{K})} \neq \emptyset$ (Lemme 2,1)), il existe $x \in U(\mathcal{F}) \cap Y_{(\widehat{K})}$ au-dessus d'un point $y$ de $Y$ (LeMMe 2,2)).

Par le Lemme 3 ci-après il existe un fermé $Z$ de $Y$ géométriquement irréductible, avec $y \in Z$ et $\operatorname{dim} Z=1$. Soit $L=\mathcal{R}(Z)$ ( $Z$ est muni de la structure réduite induite), le corps des constantes de $L$ est alors purement inséparable sur $K$ [Gro, chapitre IV, proposition 4.5.9, p. 62]. Soit $g_{i}$ la restriction de $f_{i}$ à $Z$. Comme $\left\{(K,|\cdot|) ; \mathcal{C}_{L} ; g_{1}, \ldots, g_{r}\right\}$ satisfait la propriété de Skolem, il suit du Corollaire $1 \mathrm{du}$ Lemme 2 que $\left\{(K,|\cdot|) ; Z ; g_{1}, \ldots, g_{r}\right\}$ satisfait la propriété de Skolem. Ceci veut dire qu'il existe $z \in Z$ tel que $g_{i} \in \mathcal{O}_{Z, z}$ et que $\left|\sigma\left(g_{i}(z)\right)\right| \leq 1$ pour tout $K$-homomorphisme $\sigma:\left(\mathcal{O}_{Z, z} / \mathfrak{M}_{z}\right) \rightarrow \widehat{K}^{\text {alg }}$. Comme $\left(\mathcal{O}_{Z, z} / \mathfrak{M}_{z}\right)=$ $\left(\mathcal{O}_{Y, z} / \mathfrak{M}_{z}^{\prime}\right)$ il suit que $\left\{(K,|\cdot|) ; Y ; f_{1}, f_{2}, \ldots f_{r}\right\}$ satisfait la propriété de Skolem.

Lemme 2. - Soient $(K,|\cdot|)$ un corps valué, $X$ une variété algébrique sur $K$, géométriquement irréductible, $\mathcal{F}=\left\{f_{1}, f_{2}, \ldots, f_{r}\right\}$ une partie finie de $\mathcal{R}(X)$. On suppose $U(\mathcal{F})$ non vide. Alors :

1) L'ouvert $U(\mathcal{F})$ est Zariski dense dans $X_{(\widehat{K})}$.

2) De plus, il existe un point $y \in X_{(\widehat{K})}$ qui est au-dessus d'un point (fermé) de $X$.

Démonstration.

1) Soit $Z$ un fermé de Zariski de $X_{(\widehat{K})}$ avec $U \subset Z$ (où $U=U(\mathcal{F})$ ).

томе $116-1988-\mathrm{N}^{\circ} 1$ 
Ainsi $U_{\text {red }}$ est ouvert affinoïde de $Z^{\text {an }}$ (pour les structures réduites) et on a $\operatorname{dim} U \leq \operatorname{dim} Z^{\text {an }}, \operatorname{dim} Z^{\text {an }}=\operatorname{dim} Z$. [Fr, Théorème 2, p. 118]. Soit $x \in U$, on a

$$
\operatorname{dim} \mathcal{O}_{U, x}=\operatorname{dim} \mathcal{O}_{X_{(\widehat{K})}^{\text {an }}, x}=\operatorname{dim} \mathcal{O}_{X}{ }_{(\widehat{K})}, x=\operatorname{dim} X_{(\widehat{K})}=\operatorname{dim} X
$$

(parce que $X_{(\widehat{K})}$ est irréductible); d'autre part $\operatorname{dim} \mathcal{O}_{U, x} \leq \operatorname{dim} U$. Il suit donc que $\operatorname{dim} Z \geq \operatorname{dim} X_{(\widehat{K})}$, ce qui montre que $Z=X_{(\widehat{K})}$ puisque $X_{(\widehat{K})}$ est irréductible.

2) On peut supposer $X$ réduit (donc intègre). Il existe un ouvert affine $Y$ de $X$ non vide tel que $f_{i}$ soit régulier sur $Y$. Par 1) on a $U \cap Y_{(\widehat{K})} \neq \emptyset$, ce qui montre qu'on peut supposer $X=\operatorname{Spm} A$ et $f_{i} \in A$. Il existe $T_{1}, T_{2}, \ldots, T_{n} \in A$, algébriquement indépendants sur $K(n=\operatorname{dim} A)$, $P \in K[T]=K\left[T_{1}, \ldots, T_{n}\right], P \neq 0, a \in A$ tels que $a$ soit entier sur $K[T]$ et que $A\left[P^{-1}\right]$ soit entier (purement inséparable) sur $K\left[T, P^{-1}, a\right]$. Par 1) il existe $x_{0} \in U$ et $P \notin \mathfrak{M}_{x_{0}}$ où $\mathfrak{M}_{x_{0}}$ est le maximal de $A \otimes_{K} \widehat{K}$ associé à $x_{0}$.

Soient $\varphi: A \otimes_{K} \widehat{K} \rightarrow \widehat{K}^{\text {alg }}$ un $\widehat{K}$-homomorphisme tel que $\operatorname{ker} \varphi=\mathfrak{M}_{x_{0}}$,

$$
u_{0}+u_{1} Z+\cdots+u_{m-1} Z^{m-1}+Z^{m}=\operatorname{irr}(a, K(T), Z)
$$

(on a $u_{i} \in K[T]$ ). Comme $\varphi(P) \neq 0$, quitte à changer $P$ en $\lambda P^{\alpha}$ on peut supposer que $|\varphi(P)|=1$.

Il existe des entiers $q, r$ tels que $\left(P^{r} f_{i}\right)^{q} \in K[T, a]$ et donc $\left(P^{r} f_{i}\right)^{q}=$ $\sum_{j=0}^{m-1} v_{i j} a^{j}$ avec $v_{i j} \in K[T]$. Soit $\epsilon>0$ tel que

$$
\epsilon \max \left\{1,\left|\varphi\left(v_{i j}\right)\right|,\left|\varphi\left(a^{j}\right)\right|\right\}<1 .
$$

Il existe $\xi_{1}, \ldots, \xi_{n} \in K^{\text {alg }}$ tels que

$$
\left|\varphi\left(v_{i j}\right)-v_{i j}(\xi)\right|<\epsilon,|\varphi(P)-P(\xi)|<1,\left|\varphi\left(u_{k}\right)-u_{k}(\xi)\right|\left|\varphi(a)^{j}\right|<\epsilon^{2 m} \text {. }
$$

Alors il existe $\alpha \in K^{\text {alg }}$ tel que $\sum_{k} u_{k}(\xi) \alpha^{i}=0$ et $|\varphi(a)-\alpha|<\epsilon^{2}$.

Il existe un $K$-homomorphisme $\psi: K[T][a] \rightarrow K^{\text {alg }} \subset \widehat{K}^{\text {alg }}$ avec $\psi\left(T_{i}\right)=\xi_{i}$ et $\psi(a)=\alpha$, il suit que $|\psi(P)|=1$ et que $\left|\psi\left(\left(P^{r} f_{i}\right)^{q}\right)\right| \leq$ 1. Comme $A$ est entier sur $K[T][a]$, l'homomorphisme $\psi$ admet un prolongement à $A$ toujours noté $\psi$ et on a $\left|\psi\left(f_{i}\right)\right| \leq 1 . \operatorname{Ainsi} \operatorname{ker}\left(\psi \otimes \mathbf{1}_{\widehat{K}}\right)$ est un point de $U$ au-dessus d'un point de $X$.

Corollaire 1. - Soient $(K,|\cdot|)$ un corps valué, $X$ une variété algébrique sur $K$, géométriquement irréductible, $Y$ un ouvert non vide de $X$. Alors les propriétés suivantes sont équivalentes.

i) $\{(K,|\cdot|) ; X\}$ satisfait la propriété de Skolem,

ii) $\{(K,|\cdot|) ; Y\}$ satisfait la propriété de Skolem. 
Démonstration.

i) implique ii). Soient $f_{1}, f_{2}, \ldots, f_{r} \in \mathcal{R}(Y)=\mathcal{R}(X)$ tels que

$$
V=\left\{y \in Y_{(\widehat{K})} ; f_{i} \in \mathcal{O}_{Y_{(\widehat{K})}, y} \text { et }\left|f_{i}(y)\right| \leq 1, \text { pour } 1 \leq i \leq r\right\}
$$

soit non vide. Il existe $g_{1}, g_{2}, \ldots g_{s} \in \mathcal{R}(X)$ tels que

$$
Z \stackrel{\text { déf }}{=}\left\{x \in X ; g_{i} \in \mathcal{O}_{X, x}, 1 \leq i \leq s\right\}
$$

soit non vide et contenu dans $Y$ (bien entendu $Z$ est ouvert). Par le Lemme 2.1 il existe $y_{0} \in V \cap Z_{\widehat{K}}$; soit $\pi \in K$ avec $\left|g_{i}\left(y_{0}\right)\right| \leq|\pi|$. Soient $h_{i}=\pi^{-1} g_{i}$

$$
\begin{array}{r}
\stackrel{\text { déf }}{=}\left\{x \in X ; f_{i} \in \mathcal{O}_{X, x}, h_{j} \in \mathcal{O}_{X, x},\left|f_{i}(x)\right| \leq 1, h_{j}(x) \mid \leq 1\right. \\
\text { pour } 1 \leq i \leq r, 1 \leq j \leq s\} .
\end{array}
$$

On a $U \subset Z_{(\widehat{K})}, y_{0} \in U$, ainsi par i) il existe $x \in X$ tel que $f_{i} \in \mathcal{O}_{X, x}, h_{j} \in$ $\mathcal{O}_{X, x}$ et pour tout $K$-homomorphisme $\sigma: K(x) \rightarrow \widehat{K}^{\text {alg }}$ on a

$$
\left|\sigma\left(f_{i}(x)\right)\right| \leq 1,\left|\sigma\left(h_{j}(x)\right)\right| \leq 1 \text { pour } 1 \leq i \leq r, 1 \leq j \leq s .
$$

Or $x \in Z \subset Y$, ce qui montre ii).

ii) implique i) : résulte clairement de la partie 1) du Lemme 2.

Corollaire 2. - Soient $(K,|\cdot|)$ un corps valué, $X$ une variété algébrique sur $K$, géométriquement irréductible,

$$
S=\left\{x \in X_{(\widehat{K})} \text { tel qu'il existe un point (fermé) de } X \text { au-dessous de } x\right\} \text {. }
$$

Alors $S$ est dense dans $X_{(\widehat{K})}$ (pour la topologie de $X_{(\widehat{K})}$ induite par celle de $\widehat{K})$. (Voir [Pz, § 3, Corollaire 1]).

Lemme 3. - Soient $K$ un corps infini, $X$ une variété algébrique sur $K$, géométriquement irréductible, avec $\operatorname{dim} X \geq 1$ et $x \in X$ un point fermé. Alors il existe un fermé $Y$ de $X$, de dimension 1 , géométriquement irréductible, avec $x \in Y$.

Démonstration. - Soit $n=\operatorname{dim} X$, si $n=1$ il n'y a rien à montrer. Supposons $n \geq 2$.

$\alpha$ ) Supposons $X$ affine, et le lemme montré pour les variétés $Z$ avec $\operatorname{dim} Z \leq n-1$.

$$
\text { TOME } 116-1988-\mathrm{N}^{\circ} 1
$$


Soient $P \supset X$ une variété projective dont $X$ est ouvert dense, $\pi: P^{\prime} \rightarrow$ $P$ la variété obtenue en faisant éclater le point $\{x\}$ [Gro, chapitre II, définition 8.1.3, p. 153]. Comme $\pi$ est projectif (idem définition 8.1.3), on a $P^{\prime}$ qui est projectif. Comme $\pi$ est birationnel [Gro, chapitre II, proposition $8.1 .4 \mathrm{ii})$, p. 154] on a $P^{\prime}$ qui est géométriquement irréductible. Enfin $\pi^{-1}(\{x\})$ est un fermé de $P^{\prime}$ avec $\operatorname{dim} \pi^{-1}(\{x\})=n-1$ [Gro, chapitre IV, lemme 19.4.2, p. 198].

Soient $f: P^{\prime} \rightarrow \mathbf{P}_{K}^{N}$ une immersion fermée, $\operatorname{Gr}(1, N)$ la Grassmannienne des hyperplans de $\mathbf{P}_{K}^{N}$. Il existe une partie $S$ de $\operatorname{Gr}(1, N)(K)$, Zariski dense dans $\operatorname{Gr}(1, N)$ telle que pour tout $V \in S$, le fermé $f^{-1}(V)$ de $P^{\prime}$ soit géométriquement irréductible [Jo, corollaire $6.11_{3}$, p. 89]. Il suit de cela qu'il existe $V \in \operatorname{Gr}(1, N)(K)$ avec

$$
\emptyset \neq f^{-1}(V) \cap \pi^{-1}(\{x\}) \varsubsetneqq f^{-1}(V) \quad \text { et } \quad \operatorname{dim}\left(f^{-1}(V)\right)=\operatorname{dim} P^{\prime}-1 .
$$

Comme $\pi$ est projectif, donc fermé, on a $\pi\left(f^{-1}(V)\right)$ qui est fermé; comme $\pi: P^{\prime}-\pi^{-1}(\{x\}) \rightarrow P-\{x\}$ est un isomorphisme il suit que $\pi\left(f^{-1}(V)\right)$ est géométriquement irréductible de dimension $n-1$ et que $x \in \pi\left(f^{-1}(V)\right)$. Cela implique que $\pi\left(f^{-1}(V)\right) \cap X$ est un fermé de $X$ contenant $x$, géométriquement irréductible et de dimension $n-1$. Ainsi par notre supposition $\alpha)$, il existe un fermé $Y$ de $\pi\left(f^{-1}(V)\right) \cap X$, contenant $x$, de dimension 1 et géométriquement irréductible. Ce qui montre $\alpha$ ).

$\beta)$ Le cas général. - Soit $x \in U \subset X$ un ouvert affine, par $\alpha$ ) il existe $x \in Z \subset U$ un fermé de dimension 1, géométriquement irréductible. Il suit que $\bar{Z}$ l'adhérence de $Z$ dans $X$ satisfait le lemme.

ThÉORÈmE 2. - Soient $(K,|\cdot|)$ un corps valué, $L / K$ un corps de fonctions d'une variable dont le corps des constantes $K_{1}$ est purement inséparable sur $K, \mathcal{C}_{L}$ l'unique courbe sur $K$, non singulière projective telle que $\mathcal{R}\left(\mathcal{C}_{L}\right)=L$. Alors les propriétés suivantes sont équivalentes.

i) Le couple $\left\{(K,|\cdot|) ; \mathcal{C}_{L}\right\}$ satisfait la propriété de Skolem (locale).

ii) Pour toute valeur absolue $|\cdot|_{1}$ sur $L$, prolongeant $|\cdot|$, telle que $(\overline{L,|\cdot|}) /(\overline{K,|\cdot|})$ soit un corps de fonctions à une variable, il existe $T \in L-K_{1}$ tel que $|\cdot|_{1}$ soit l'unique prolongement à $L$ de la valeur absolue de Gauss sur $K(T)$ associée $\grave{a}|\cdot|$ et $\grave{a} T$.

iii) Pour toute famille $\left\{|\cdot|{ }_{j}\right\}_{1 \leq j \leq s}$ de valeurs absolues sur L prolongeant $|\cdot|$, telle que $\left(\overline{L,|\cdot|_{j}}\right) /(\overline{K,|\cdot|})$ soit un corps de fonctions d'une variable, il existe $T \in L-K_{1}$ tel que $\left\{|\cdot|_{j}\right\}_{1 \leq j \leq s}$ soient exactement les prolongements à $L$ de la valeur absolue de Gauss sur $K(T)$ associée à $|\cdot|$ et $\grave{a} T$.

Démonstration.

i) implique ii). Soit $Z_{1} \in L-K_{1}$ tel que $|\cdot|_{1}$ soit un prolongement à 
$L$ de la valeur absolue de Gauss sur $K\left(Z_{1}\right)$ associée à $|\cdot|$ et à $Z_{1}$. Soient $r: \mathcal{C}_{L \widehat{K}}^{\text {an }} \rightarrow \mathcal{E}$ la réduction analytique associée à $Z_{1}$ ( $(1.2$, proposition 1$)$, $\mathcal{E}_{1}$ la composante irréductible de $\mathcal{E}$ correspondant $|\cdot|_{1}$.

Soient $R$ (resp. $R_{1}$ ) la clôture intégrale de $K\left[Z_{1}\right]$ (resp. $\left.\widehat{K}\left[Z_{1}\right]\right)$ dans $L$ (resp. $L \widehat{K}$ ). Notons $R \widehat{K}$ la sous- $\widehat{K}$-algèbre de $L \widehat{K}$ engendrée par $R$; c'est aussi $\left(R \otimes_{K} \widehat{K}\right)_{\text {red }}$. Comme $\operatorname{Fr}(R \widehat{K})=\operatorname{Fr}\left(R_{1}\right)$ il existe $P \in \widehat{K}^{\circ}\left[Z_{1}\right]$ tel que $R \widehat{K}\left[P^{-1}\right]=R_{1}\left[P^{-1}\right]$ et $\bar{P} \neq 0$. Soient $U=U\left(Z_{1}\right)$, on a $\bar{R}_{1}=\mathcal{O}_{\mathcal{E}}(r(U))$ et donc $\bar{R}_{1}\left[\bar{P}^{-1}\right]=\bar{R}\left[\bar{P}^{-1}\right]=\mathcal{O}_{\mathcal{E}}(r(U))\left[\bar{P}^{-1}\right](\S 1.2$, Proposition 1$)$.

Soit $W$ un ouvert principal non vide de $r(U)$ avec $W \subset D(\bar{P}) \cap \mathcal{E}_{1}$. Ainsi il existe $f \in R$ avec $\|f\|=1$ et $W=D(\bar{f})$. On a donc

$$
r^{-1}(W)=\left\{y \in \mathcal{C}_{L \widehat{K}} ; f^{-1}, Z_{1} \in \mathcal{O}_{\mathcal{C}_{L \widehat{K}}, y},\left|f^{-1}(y)\right| \leq 1,\left|Z_{1}(y)\right| \leq 1\right\} .
$$

Comme $r^{-1}(W) \neq \emptyset$, et $\mathcal{C}_{L \widehat{K}}=\left(\left(\left(\mathcal{C}_{L}\right)_{(\widehat{K})}\right)_{\text {red }}\right)^{\prime}$, il existe $x \in \mathcal{C}_{L}$ avec

$$
f^{-1}, Z_{1} \in \mathcal{O}_{\mathcal{C}_{L}, x}, \quad \mid \sigma\left(f^{-1}(x)|\leq 1, \quad| \sigma\left(Z_{1}(x)\right) \mid \leq 1\right.
$$

pour tout $K$-homomorphisme $\sigma: K(x) \rightarrow \widehat{K}^{\text {alg }}$. Cela veut dire que pour tout $y_{i} \in \mathcal{C}_{L \widehat{K}}$ au-dessus de $x$ on a

$$
f^{-1}, Z_{1} \in \mathcal{O}_{\mathcal{C}_{L \widehat{K}}, y_{i}}
$$

et $\left|f^{-1}\left(y_{i}\right)\right| \leq 1,\left|Z_{1}\left(y_{i}\right)\right| \leq 1$, i.e., $y_{i} \in r^{-1}(W)$.

Ainsi ii) du THÉORÈME 1 est satisfait pour $\left(L / K,|\cdot|_{1}\right)$. Il suit donc de ce même théorème qu'il existe $T \in L-K_{1}$ tel que $|\cdot|_{1}$ soit l'unique prolongement à $L$ de la valeur absolue de Gauss sur $K(T)$ associée à $|\cdot|$ et à $T$.

ii) implique iii) n'est autre que le ThÉORÈme 1 et son CoROllaIRE 1.

iii) implique i). Soient $f_{1}, f_{2}, \ldots, f_{r} \in L$, on suppose qu'il existe $z \in\left(\mathcal{C}_{L}\right)_{(\widehat{K})}$ tel que $f_{i} \in \mathcal{O}_{\mathcal{C}_{L(\widehat{K})}, z}$ et $\left|f_{i}(z)\right| \leq 1$ pour $1 \leq i \leq r$.

Soient $U=\left\{y \in \mathcal{C}_{L \widehat{K}} ; f_{i} \in \mathcal{O}_{\mathcal{C}_{L \widehat{K}}, y},\left|f_{i}(y)\right| \leq 1\right.$, pour $\left.1 \leq i \leq r\right\}$. Comme $\mathcal{C}_{L \widehat{K}}=\left(\left(\left(\mathcal{C}_{L}\right)_{(\widehat{K})}\right)_{\mathrm{red}}\right)^{\prime}$, i.e., la normalisation de $\left(\left(\mathcal{C}_{L}\right)_{(\widehat{K})}\right)_{\mathrm{red}}$ il suit de ce qui précède que $U \neq \emptyset$. Ainsi $U$ est un admissible non vide de $\left(\mathcal{C}_{L \widehat{K}}\right)^{\text {an }}(c f . \S 1.1 .2)$. Si $U=\mathcal{C}_{L \widehat{K}}$ la propriété de Skolem est satisfaite pour le triplet $\left\{(K,|\cdot|) ; \mathcal{C}_{L \widehat{K}} ; f_{1}, f_{2}, \ldots, f_{r}\right\} ;$ sinon l'admissible $U$ est affinoïde (cf. $\S 1.1 .2$ ), ce que l'on supposera désormais.

Chaque composante irréductible de $\bar{U}^{c}$ (la réduction canonique de $U$ ) définit une valeur absolue $|\cdot|_{j}$ sur $L$ telle que $\left(\overline{L,|\cdot|_{j}}\right) /(\overline{K,|\cdot|})$ soit un corps de fonctions d'une variable ( $\$ 1.2$, proposition 1$)$. Soit $\left\{|\cdot|_{j}\right\}_{1 \leq j \leq s}$ cet ensemble de valeurs absolues, $\|\cdot\| \stackrel{\text { déf }}{=} \max _{j}|\cdot|_{j}$, on a donc $\left\|f_{i}\right\| \leq 1$.

TOME $116-1988-\mathrm{N}^{\circ} 1$ 
Par iii) il existe $T \in L-K$ tel que $\left\{|\cdot|_{j}\right\}_{1 \leq j \leq s}$ soient exactement l'ensemble des prolongements à $L$ de la valeur absolue de Gauss sur $K(T)$ associée à $|\cdot|$ et à $T$. Il existe $P_{i}(T) \in K[T]$ tel que $P_{i}(T) f_{i}$ soit entier sur $K[T]$ et que $\left\|P_{i}(T)\right\|=1$. Soient $r: \mathcal{C}_{L \widehat{K}}^{\text {an }} \rightarrow \mathcal{E}$ la réduction analytique associée à $T(\S 1.2$, proposition 1$), P=\prod_{i} P_{i}$

$$
U(T)=\left\{y \in \mathcal{C}_{L \widehat{K}} ; T \in \mathcal{O}_{\mathcal{C}_{L \widehat{K}}, y} \text { et }|T(y)| \leq 1\right\},
$$

$D(\bar{P})$ l'ouvert principal de $r(U(T))$ associé à $\bar{P}$. Alors on a $r^{-1}(D(\bar{P})) \neq \emptyset$, $f_{i} \in \mathcal{O}_{\mathcal{C}_{L \widehat{K}}, y}$ et $\left|f_{i}(y)\right| \leq 1$ pour tout $y \in r^{-1}(D(\bar{P}))$.

Soit $\mathcal{E}_{1}$ la composante irréductible de $\mathcal{E}$ qui correspond à $|\cdot|_{1}$, on a $\mathcal{E}_{1} \cap D(\bar{P}) \neq \emptyset$, soit $W$ un ouvert non vide $\mathcal{E}$ avec $W \subset \mathcal{E}_{1} \cap D(\bar{P})$. Comme iii) est satisfait pour $\left\{|\cdot|_{1}\right\}$ il suit que i) du ThÉorème 1 l'est aussi, donc iii) du ThÉorème 1 est vérifié. Donc il existe $x \in \mathcal{C}_{L}$ tel que pour tout $y_{i} \in \mathcal{C}_{L \widehat{K}}$ au-dessus de $x$, on ait $y_{i} \in r^{-1}(W) \subset r^{-1}(D(\bar{P}))$. Ceci veut dire que $f_{i} \in \mathcal{O}_{\mathcal{C}_{L}, x}$ et que $\left|\sigma\left(f_{i}(x)\right)\right| \leq 1$ pour tout $K$-homomorphisme $\sigma: K(x) \rightarrow \widehat{K}^{\text {alg }}$.

Il suit que la famille $\left\{(K,|\cdot|) ; \mathcal{C}_{L} ; f_{1}, \ldots, f_{r}\right\}$ satisfait la propriété de Skolem (locale). Ainsi i) est montré.

Corollaire 1. - Soit $(K,|\cdot|)$ un corps valué. Alors les propriétés suivantes sont équivalentes.

i) Le corps valué $(K,|\cdot|)$ satisfait la propriété de Skolem (locale),

ii) pour tout corps de fonctions à une variable $L / K$ dont le corps des constantes est purement inséparable sur $K$ et pour toute famille $\left\{|\cdot|_{j}\right\}_{1 \leq j \leq s}$ de valeurs absolues sur $L$ prolongeant $|\cdot|$, telle que le quotient $(\overline{L,|\cdot| j}) /(\overline{K,|\cdot|})$ soit un corps de fonctions à une variable, la propriété ii) $d u$ THÉORÈme 2 est satisfaite.

Démonstration. - C'est le Théorème 2 et la Proposition 5.

Corollaire $2[\mathrm{Ma}, \mathrm{Oh}]$. - Soient $\left(K,|\cdot|_{0}\right)$ un corps valué, $K(X)$ le corps des fractions rationnelles à une variable sur $K,|\cdot|$ une valeur absolue sur $K(X)$ qui prolonge $|\cdot|_{0}$ et telle que $(\overline{K(X),|\cdot|})$ soit un corps de fonctions d'une variable sur $\left(\overline{K,|\cdot|_{0}}\right)$. Alors il existe $T \in K(X)-K$ tel $q u e|\cdot|$ soit l'unique prolongement à $K(X)$ de la valeur absolue de Gauss sur $K(T)$ associée à $T$ et $|\cdot|_{0}$.

Démonstration. - C'est une conséquence du Corollaire 1 de la Proposition 4 et du ThÉorème 2.

Remarque 1. - Soit $g \geq 1$ un entier. Alors il existe un corps valué $(K,|\cdot|)$ et une courbe $\mathcal{C}$ projective non singulière, géométriquement intègre sur $K$ telle que $\{(K,|\cdot|) ; \mathcal{C}\}$ ne satisfasse pas la propriété de Skolem. 
Rappelons tout d'abord qu'une courbe de genre zéro satisfait la propriété de Skolem (Proposition $4^{\prime}, \S 3$ ). La construction d'un corps valué $(K,|\cdot|)$ et d'une courbe $\mathcal{C}$ sur $K$ qui ne satisfait pas la propriété de Skolem nous a été suggérée par M. RAYNAUD et aussi aimablement commentée par L. Moret-BAILly. Dans [Pz], §4, il y a une démonstration complète de cela.

Pour $g=1$ nous pouvons présenter l'exemple. Soient $K=\mathbb{C}(t)$ le corps des fractions rationnelles sur le corps des complexes, $|\cdot|$ une valeur absolue sur $K$ défini par $0<|t|<1$ et $|\lambda|=1$ pour tout $\lambda \in \mathbb{C}-\{0\}$, $K^{0}$ l'anneau de valuation de $(K,|\cdot|), k=\mathbb{C}$ le corps résiduel de $(K,|\cdot|)$ (bien entendu le corps résiduel n'est pas algébrique sur un corps fini : $c f$. théorème [Ru], [Ro2], [Mo-Ba, Szp], [Mo-Ba]).

Soient

$$
A=\frac{K^{0}[X, Y, Z]}{\left(Y^{2} Z-X(X-Z)(X-t Z)\right)}=K^{0}[x, y, z]
$$

la $K^{0}$-algèbre graduée par $\operatorname{deg} x=\operatorname{deg} y=\operatorname{deg} z=1, \mathfrak{X}=\operatorname{Proj} A$, $\mathfrak{X}_{K}=\operatorname{Proj}\left(A \otimes_{K^{0}} K\right)$ (la fibre générique de $\left.\mathfrak{X}\right), \mathfrak{X}_{k}=\operatorname{Proj}\left(A \otimes_{K^{0}} k\right.$ ) (la fibre spéciale de $\mathfrak{X})$. Il existe un point fermé $a \in \mathfrak{X}_{k}$ tel que $\{a\} \neq \mathfrak{X}_{k} \cap \overline{\{p\}}$ pour tout point fermé $p \in \mathfrak{X}_{K}$ (ici $\overline{\{p\}}$ désigne l'adhérence de $\{p\}$ dans $\mathfrak{X}$ ).

Expliquons brièvement l'existence de $a \in \mathfrak{X}_{k}$. Par un théorème de LANG-NÉRON [La, théorème 2 , p. 139] on sait que $\mathfrak{X}_{K}(K)$ est un groupe de type fini ( $\mathfrak{X}_{K}$ est une courbe elliptique), donc dénombrable. Par ailleurs $\mathfrak{X}_{k}(k)$ est un groupe non dénombrable puisque quotient de $\mathbb{C}$ par un réseau ( $\mathfrak{X}_{k}$ est une courbe elliptique).

Ainsi il existe $a \in \mathfrak{X}_{k}(k)$ tel que $\{d a\} \neq \mathfrak{X}_{k} \cap \overline{\{p\}}$ pour tout point $p$ de $\mathfrak{X}_{k}(K)$ et pour tout entier $d \geq 1$ ( $d a$ signifie l'addition dans la courbe elliptique $\mathfrak{X}_{k}$ ). Alors en utilisant que $\mathfrak{X}_{K}$ et $\mathfrak{X}_{k}$ sont des courbes elliptiques on peut montrer que $\{a\} \neq \mathfrak{X}_{k} \cap \overline{\{p\}}$ pour tout point fermé $p$ de $\mathfrak{X}_{K}$. Le point $a$ de $\mathfrak{X}_{k}(k)$ a pour coordonnées projectives $[u, v, 1], u, v \in \mathbb{C}$; soient $f_{1}=t^{-1}\left(z^{-1} x-u\right), f_{2}=t^{-1}\left(z^{-1} y-v\right)$, on a donc $f_{i} \in \mathcal{R}\left(\mathfrak{X}_{K}\right)$ pour $i=1,2$, alors on peut montrer que $\left\{(K,|\cdot|) ; \mathfrak{X}_{K} ; f_{1}, f_{2}\right\}$ ne satisfait pas la propriété de Skolem; sinon il existerait un point fermé $q \in \mathfrak{X}_{K}$ avec $\{a\}=\mathfrak{X}_{k} \cap \overline{\{q\}}$.

Cela se traduit en termes de prolongement de la valeur absolue de Gauss. Soit $T=f_{1} \in L=\mathcal{R}\left(\mathfrak{X}_{K}\right)$, alors la valeur absolue de Gauss sur $K(T)$ associée à $T$ et à $|\cdot|$ admet exactement deux prolongements à $L$ que l'on note $|\cdot|_{1}$ et $|\cdot|_{2}$.

Ce qui précède montre qu'il n'existe pas $T_{1}$ transcendant sur $K$ tel que $|\cdot|_{1}$ (resp. $\left.|\cdot|_{2}\right)$ soit l'unique prolongement à $L$ de la valeur absolue de Gauss sur $K\left(T_{1}\right)$ associée à $T_{1}$ et à $|\cdot|$.

TOME $116-1988-\mathrm{N}^{\circ} 1$ 
Remarque 2. Les notions "Träge" chez E. LAMPrecht et P. RoQUetTE [La2], [Ro1].

Soient $(L / K,|\cdot|)$ un corps de fonctions d'une variable muni d'une valeur absolue discrète (i.e., $\left|L^{\times}\right| \simeq \mathbf{Z}$ ) tel que $\bar{L} / \bar{K}$ soit un corps de fonctions d'une variable.

Selon LAmprecht [La2], $(L / K,|\cdot|)$ satisfait la propriété "Träge" s'il existe $T \in L-K$ avec $|T|=1, T$ résiduellement transcendant sur $\bar{K}$ et $[L: K(T)]=[\bar{L}: \overline{K(T)}]$.

Selon RoquetTe [Ro1], $(L / K,|\cdot|)$ satisfait la propriété "Träge" si pour tout sous- $K$-espace vectoriel $E$ de $L$ de dimension finie on a $\operatorname{dim}_{K} E=$ $\operatorname{dim}_{\bar{K}} \bar{E}$.

On peut montrer que "Träge" selon LAMPRECHT implique "Träge" selon Roquette. En revanche l'autre implication est fausse. En effet le corps des fonctions $\left(L / K,|\cdot|_{1}\right)$ de la REMARQUE 1 satisfait la propriété "Träge" selon RoQUETTE et non celle selon LAMPRECHT.

En revanche si $\left\{(K,|\cdot|) ; \mathcal{C}_{L}\right\}$ satisfait la propriété de Skolem (locale) les deux notions de "Träge" sont équivalentes.

\section{BIBLIOGRAPHIE}

[Bo1] Bosch (S.). - $K$-affinoid Tori, Math. Ann, t. 192, 1971, p. 1-16.

[Bo2] BosCH (S.). - Zur Kohomologietheorie rigid analytisher Raüme, Manuscripta Math., t. 20, 1977, p. 1-27.

[BGR] Bosch (S.), Güntzer (U.), Remmert (R.). - Non-Archimedean Analysis. Springer-Verlag, 1984 .

[Bki] BourbaKi (N.). - Algèbre, chap. V. - Paris, Hermann, 1981.

[De1] Deuring (M.). - Reduktion algebraischer Funktionenkörper nach Primdivisorem des Konstantenkörpers, Math. Z., t. 47, 1942, p. 643-654.

[De2] Deuring (M.). - Die Zetafunktion einer algebraischen Kurve vom. Geschlechte Eins. II. Nach. Akad. Wiss. Göttingen, Math-Phys., t. Kl, 1955, p. $13-42$.

[Fr] Fresnel (J.). - Géométrie analytique rigide, Université de Bordeaux I, 1984, (Polycopié).

[Fr,Ma] Fresnel (J.), MAtignon (M.). - Structure des espaces analytiques quasicompacts de dimension 1 sur un corps valué, complet, ultramétrique, Ann. Mat. Pura Appl. (4), t. 145, 1986, p. 159-210.

[Fr,vdP] Fresnel (J.), v. der Put (M.). - Géométrie analytique rigide et applications. Birkhäuser, 1981 (Progr. Math., 18).

[Gro] Grothendieck (A.). - EGA, Inst. Hautes Études Sci. Publ. Math., t. 11, 1961, p. 24-28.

[Jo] JouANOLOU (J.-P.). - Théorèmes de Bertini et applications. - Birkhäuser, 1983 (Progr. Math. 42).

BULLETIN DE LA SOCIÉTÉ MATHÉMATIQUE DE FRANCE 
[La] LANG (S.). - Fundamentals of diophantine geometry. - Springer-Verlag, 1983 .

[La1] LAMPrecht (E.). - Zur Eindeutigkeit von Funktionalprimdivisoren, Arch. Math., t. 8, 1957, p. 30-38.

[La2] Lamprecht (E.). - Restabbildungen von Divisoren I, Arch. Math., t. 8, 1957 , p. $255-264$.

[Ma1] MAtignon (M.). - Genre et genre résiduel des corps de fonctions valuées, 1987 , (Thèse, Bordeaux I).

[Ma2] Matignon (M.). - Genre et genre résiduel des corps de fonctions valués, Manuscripta Math., t. 58 (fasc. 1/2), 1987, p. 179-214.

[Ma,Oh] Matignon (M.), Онм (J.). - A structure theorem for simple transcendental extensions of valued fields, (à paraître), Proc. Amer. Math. Soc..

[Mo-Ba] MORET-BAILly (L.). - Problèmes diophantiens sur l'anneau des entiers algébriques, Séminaire de théorie des nombres de Bordeaux, [exp. $\mathrm{n}^{\circ} 22.1985^{-}$ 86]. Point entier des variétés arithmétiques, Séminaire DPP, 1985-86.

[Mo-Ba,Szp] Moret-BAILly (L.), SzPIRo (L.). - à paraître.

[Ne] NERING (E.). - Reduction of an algebraic function field modulo a prime in the constant field, Ann. of Math. (2), t. 67, 1957, p. 590-606.

[Po] POPP (H.). - Über das Verhalten des Geschlechts eines Funktionenkörpers, Math. Z., t. 106, 1968, p. 17-35.

[Pz] Polzin (M.). - Prolongement de la valeur absolue de Gauss et propriété de Skolem, (Thèse $3^{\mathrm{e}}$ cycle, Université de Bordeaux I, 1987).

[Ro1] ROQUETTE (P.). - Zur Theorie der Konstantenreduktion algebraischer Mannigfaltigkeiten, J. Reine Angew. Math., t. 200, 1958, p. 1-44.

[Ro2] ROQUETTE (P.). - Solving diophantine equations over the ring of all algebraic integers, [Atas da $8^{\mathrm{e}}$ Escola de Algebra, vol. 2], IMPA 84.

[Ru] RUMELY (R.). - Arithmetic over the ring of all algebraic integers, J. Reine Angew. Math., t. 368, 1986, p. 127-133.

[Sk] SKolem (T.). - Lösung gewisser Gleichungen in ganzen algebraischen Zahlen insbesondere in Einheiten, Skrifter Norske Videnkaps Akademi, Oslo, Math. Naturv., t. 10, 1935, p. 1-19.

TOME $116-1988-\mathrm{N}^{\circ} 1$ 